\title{
A Variational Technique for Time Consistent Tracking of Curves and Motion
}

\author{
N. Papadakis · E. Mémin
}

(C) Springer Science+Business Media, LLC 2008

\begin{abstract}
In this paper, a new framework for the tracking of closed curves and their associated motion fields is described. The proposed method enables a continuous tracking along an image sequence of both a deformable curve and its velocity field. Such an approach is formalized through the minimization of a global spatio-temporal continuous cost functional, w.r.t a set of variables representing the curve and its related motion field. The resulting minimization process relies on optimal control approach and consists in a forward integration of an evolution law followed by a backward integration of an adjoint evolution model. This latter pde includes a term related to the discrepancy between the current estimation of the state variable and discrete noisy measurements of the system. The closed curves are represented through implicit surface modeling, whereas the motion is described either by a vector field or through vorticity and divergence maps depending on the kind of targeted applications. The efficiency of the approach is demonstrated on two types of image sequences showing deformable objects and fluid motions.
\end{abstract}

Keywords Variational method - Data assimilation · Curve tracking $\cdot$ Dynamical model

\footnotetext{
N. Papadakis $(\varangle)$

Barcelona Media, Universitat Pompeu Fabra, 8 passeig de Circumval-lació, 08003 Barcelona, Spain

e-mail: nicolas.papadakis@gmail.com

E. Mémin

CEFIMAS, Centro de Física y Matemática de América del Sur, Avenida Santa Fe 1145, C1059ABF, Buenos Aires, Argentina

e-mail: memin@irisa.fr
}

N. Papadakis · E. Mémin

VISTA, INRIA Rennes Bretagne-Atlantique, Campus de

Beaulieu, 35042 Rennes cedex, France

\section{Introduction}

\subsection{Motivation and Scope}

Tracking the contours and the motion of an object is an essential task in many applications of computer vision. For several reasons such a generic issue appears to be very challenging in the general case. As a matter of fact, the shape of a deformable object or even of a rigid body may change drastically when visualized from an image sequence. These deformations are due to the object's proper apparent motion or to perspective effect and 3D shape evolution. This difficulty is amplified when the object becomes partially or totally occluded during even a very short time period. Such a visual tracking is also a challenging issue when the curve of interest delineates iso-quantities transported by a fluid flow. This last case is of importance in domains such as meteorology or oceanography where one may wish to track iso-temperature, contours of cloud systems, or the vorticity of a motion field. Here, the most difficult technical aspect consists in handling the tracking of these features in a consistent way with respect to appropriate physical conservation laws. Another serious difficulty comes from the dimensionality of the state variables. In contexts involving for instance geophysical flows, the targeted curve which represents clouds, sea color or temperature may exhibit high topological changes and presents unsteady irregularities over time. Such behaviors make difficult the use of a reduced parametric description of the curves. They can be only described in spaces of very large dimension. In addition, the fluid motion of the regions enclosed by such curves may only be accurately described by dense motion fields. As a result, the joint tracking of a curve and its underlying motion requires to handle a state space of huge dimension. This curse of dimensionality re- 
mains true for general unknown natural objects (either rigid or deformable) observed in complex environments.

\subsection{Related Works and Their Limitations}

This context makes difficult the use of recursive Bayesian filters such as the particle filter [7], since stochastic sampling in large state spaces is usually inefficient. Even if such a filter has recently been used for fluid motion tracking [21] or curve tracking [52], this kind of techniques are only applicable when the unknown state can be described on a reduced set of basis functions. Furthermore, coping with a coupled tracking of curves and motion fields augments significantly the problem complexity. For curve tracking issues, numerous approaches based on the level set representation have been proposed [19, 24, 28, 37, 40, 45, 50, 54]. All these techniques describe the tracking as successive $2 \mathrm{D}$ segmentation processes sometimes enriched with a motion based propagation step. Segmentation techniques on spatio-temporal data have also been proposed [4, 24]. Since level sets methods do not introduce any temporal consistency related to a given dynamical law-i.e. a tracking process-, they are quite sensitive to noise [38] and exhibit inherent temporal instabilities.

Implausible growing/decreasing or merging/splitting can not be avoid without introducing hard ad hoc constraints or some statistical knowledges on the shape $[17,20,33$, 49] and, as a consequence, dedicates the process to very specific studies. Besides, general level set approaches can hardly handle occlusions of the target or cope with severe failures of the image measurements (for instance a complete loss of image data, a severe motion blur, high saturation caused by over exposure or failure of the low level image detectors). Moreover, in the context of fluid motion, a prior learning of the "fluid object" shape is in essence almost impossible and the inclusion of fluid dynamical laws related to the Navier-Stokes equation is essential to provide coherent physical plausible solution. An other idea, proposed in [5] or [60], consists in estimating the deformation allowing the transformation of the tracked object from one frame to the next frame. However, even if they allow a continuous trajectory of the tracked object to be reconstructed, in their current version, these approaches do not enable to stick the trajectory of a given dynamic.

In [56], an approach based on a group action mean shape has been used in a moving average context. Contrary to previous methods, this approach introduces, through the moving average technique, a kind of tracking process. This tracking is restricted to simple motions and does not allow the introduction of a complex dynamical law defined through differential operators. The explicit introduction of a dynamic law in the curve evolution law has been considered in [40]. However, the proposed technique needs a complex detection mechanism to cope with occlusions. Other methods, reminiscent to stochastic filtering recipes, have been proposed $[17,22,27,41,51,52]$ to operate such a tracking. However, in order to face the associated state space dimension, these methods proposed solving the problem on a lower dimensional state space or relying on a suboptimal strategy which neglects the influence of the process history or incorporates only simple discrete dynamics eventually learned from examples. The corresponding dynamics do not take into account the continuous nonlinear processes that may underly general shape deformations. Suboptimal strategy relying on a unique forward predictive step can not guaranty a global dynamical coherency of the whole trajectory. Even worse, if at a given instant, bad observation lead to an erroneous estimation the whole process diverges as the propagation will enforce in the next frame a false prediction. In order to provide an estimation that is robust to particular failures and to enforce a global dynamical consistency it is mandatory to rely on the complete trajectory of the state variable of interest.

\subsection{Contribution}

In this paper, we propose a technique which enables to handle both the tracking of closed curves and the underlying motion field transporting this curve. The approach is related to variational data assimilation technique used for instance in meteorology [6, 32, 57]. This method, based on optimal control theory [36] includes a forward-backward integration process on the whole image sequence that allows the enforcement of a global temporal consistency of the estimates. Such a technique enables, in the same spirit as a Kalman filter, a temporal smoothing along the whole image sequence. It combines a dynamical evolution law of state variables representing the target of interest with the whole set of available measurements related to this target. Unlike Bayesian filtering approaches which aim at estimating a probability law of the stochastic process associated to the feature of interest, variational assimilation has the advantage of handling state spaces of high dimension. From the motion analysis point of view, such framework enables to incorporate a dynamical consistency along the image sequence.

The framework we propose provides an efficient technique to incorporate general dynamical constraints into a coupled motion and segmentation process. As it is demonstrated in this paper, the method is particularly well suited to the analysis of fluid motion and deformations. This technique could also be useful for batch video processing. We provide some results toward this direction even if efficient answers for such applications would need much more works. 
1.4 Outline of the Paper

The paper is organized as follows. After a description of the data assimilation technique in Sect. 2, we introduce the proposed curve tracking method in Sect. 3. The method is then extended for a joint motion and object tracking in Sect. 4 . Two kinds of applications are studied here: fluid motion tracking in Sect. 4.1 and natural object tracking in Sect. 4.2. As it will be demonstrated in the experimental sections, such a technique enables to handle naturally noisy data and complete loss of image data over long time periods without resorting to complex mechanisms. This paper extends a conference paper which focuses on contours tracking [47].

\section{Variational Tracking Formulation}

In this section, we first describe the general framework proposed for tracking problems. It relies on variational data assimilation concepts $[6,32,57]$ proposed for the analysis of geophysical flows. For a sake of clarity, we will first present a simplified formulation where the state variable of interest obeys to a perfect evolution law. Let us note that this situation corresponds to the most usual model used in geophysical domain for data assimilation. This section will also be for us a mean to define properly the basic ingredients of the framework.

\subsection{Data Assimilation with Perfect Model}

Direct Evolution Model Let the state space $\mathcal{V}$ be an Hilbert space identified to its dual space. Noting $X \in \mathcal{W}\left(t_{0}, t_{f}\right)$ the state variable representing the feature of interest, which is assumed to live in a functional space $\mathcal{W}\left(t_{0}, t_{f}\right)=\{X \mid$ $\left.X \in L^{2}\left(t_{0}, t_{f} ; \mathcal{V}\right), \partial_{t} X \in L^{2}\left(t_{0}, t_{f} ; \mathcal{V}\right)\right\}$ and assuming that the evolution in time range $\left[t_{0} ; t_{f}\right]$ of the state is described through a (nonlinear) differential model $\mathbb{M}: \mathcal{V} \times$ ]$t_{0}, t_{f}[\rightarrow \mathcal{V}$, we get the following direct problem:

For a given $\eta \in \mathcal{V}$, let us define $X \in \mathcal{W}\left(t_{0}, t_{f}\right)$ such that:

$\left\{\begin{array}{l}\partial_{t} X(t)+\mathbb{M}(X(t), t)=0, \\ X\left(t_{0}\right)=X_{0}+\eta\end{array}\right.$

This system gathers an evolution law and an initial condition of the state variable. It is governed by a control variable $\eta \in \mathcal{V}$, identified here to the inaccuracy on the initial condition. The control could also be defined on model's parameters [32]. The direct problem (1) will be assumed to be well posed, which means that we first assume that the application $\mathcal{V} \rightarrow \mathcal{V}: \eta \mapsto X(\eta, t)$ is differentiable $\forall t \in] t_{0}, t_{f}[$ and secondly that given $\eta \in \mathcal{V}$ and $t_{f}>t_{0}$, there exists a unique function $X \in \mathcal{W}\left(t_{0}, t_{f}\right)$ solution of problem (1) and that this solution depends continuously on $\eta$ (i.e: $\mathcal{V} \rightarrow \mathcal{V}:$ $\eta \mapsto X(\eta, t)$ is continuous $\forall t \in] t_{0}, t_{f}[)$. Let us also assume that some measurements (also called observations) $Y \in \mathcal{O}$ of the state variable components are available. These observations may live in a different space (a reduced space for instance) from the state variable. We will nevertheless assume that there exists a (nonlinear) observation operator $\mathbb{H}: \mathcal{V} \rightarrow \mathcal{O}$, that goes from the variable space to the observation space.

Cost Function Let us define an objective function $J$ : $\mathcal{V} \rightarrow \mathbb{R}$ measuring the discrepancy between a solution associated to an initial state control variable of high dimension and the whole sequence of available observations as:

$J(\eta)=\frac{1}{2} \int_{t_{0}}^{t_{f}}\|Y-\mathbb{H}(X(\eta, t), t)\|_{R}^{2} d t+\frac{1}{2}\|\eta\|_{B}^{2}$.

The overall problem that we are facing consists in finding the control variable $\eta \in \mathcal{V}$ that minimizes the cost function $J$. Norms $\|\cdot\|_{R}$ and $\|\cdot\|_{B}$ are respectively associated to the scalar products $\left\langle R^{-1} \cdot, \cdot\right\rangle>_{\mathcal{O}}$ and $\left\langle B^{-1} \cdot, \cdot\right\rangle_{\mathcal{V}}$, where $R$ and $B$ are symmetric positive defined endomorphisms of $\mathcal{V}$. In our applications, $R$ and $B$ are respectively called, with some abuse of language, the observation covariance matrix and the initialization covariance matrix. They enable to weight the deviations from the true initial state to the given initial condition and to quantify the exactitude of the observation model.

Differential Model In order to compute the partial derivatives of the cost function with respect to the control variable, system (1) is differentiated with respect to $\eta$ in the direction $\delta \eta$. The following differential model is obtained:

Given $\eta \in \mathcal{V}, X(t)$ a solution of (1)

and a perturbation $\delta \eta \in \mathcal{V}$,

$d X=\frac{\partial X}{\partial \eta} \delta \eta \in \mathcal{W}\left(t_{0}, t_{f}\right)$ is such that:

$\left\{\begin{array}{l}\partial_{t} d X(t)+\left(\partial_{X} \mathbb{M}\right) d X(t)=0, \\ d X\left(t_{0}\right)=\delta \eta\end{array}\right.$

In this expression, the tangent linear operator $\left(\partial_{X} \mathbb{M}\right)$ is defined as the Gâteaux derivative of the operator $\mathbb{M}$ at point $X$ :

$\left(\partial_{X} \mathbb{M}\right) d X(t)=\lim _{\beta \rightarrow 0} \frac{\mathbb{M}(X(t)+\beta d X(t))-\mathbb{M}(X(t))}{\beta}$.

The tangent linear operator $\left(\partial_{X} \mathbb{H}\right)$ associated to $\mathbb{H}$ may be defined similarly. Differentiating now the cost function (2) with respect to $\eta$ in the direction $\delta \eta$ leads to:

$\left\langle\frac{\partial J}{\partial \eta}, \delta \eta\right\rangle_{\mathcal{V}}=-\int_{t_{0}}^{t_{f}}\left\langle Y-\mathbb{H}(X),(\partial X \mathbb{H}) \frac{\partial X}{\partial \eta} \delta \eta\right\rangle_{R} d t$ 


$$
\begin{aligned}
& +\left\langle X\left(t_{0}\right)-X_{0}, \delta \eta\right\rangle_{B} \\
= & -\int_{t_{0}}^{t_{f}}\left\langle\left(\partial_{X} \mathbb{H}\right)^{*} R^{-1}(Y-\mathbb{H}(X)), \frac{\partial X}{\partial \eta} \delta \eta\right\rangle_{\mathcal{V}} d t \\
& +\left\langle B^{-1}\left(X\left(t_{0}\right)-X_{0}\right), \delta \eta\right\rangle_{\mathcal{V}}
\end{aligned}
$$

where $\left(\partial_{X} \mathbb{H}\right)^{*}$, the adjoint operator of $\left(\partial_{X} \mathbb{H}\right)$, is defined by the scalar product:

$\forall x \in \mathcal{V}, \forall y \in \mathcal{O} \quad\left\langle\left(\partial_{X} \mathbb{H}\right) x, y\right\rangle_{\mathcal{O}}=\left\langle x,\left(\partial_{X} \mathbb{H}\right)^{*} y\right\rangle_{\mathcal{V}}$

Adjoint Evolution Model In order to estimate the gradient of the cost function $J$, a first numerical brute force approach consists in computing the functional gradient through finite differences:

$$
\nabla_{u} J \simeq\left[\frac{J\left(\eta+\epsilon e_{k}\right)-J(\eta)}{\epsilon}, k=1, \ldots, p\right],
$$

where $\epsilon \in \mathbb{R}$ is an infinitesimal perturbation and $\left\{e_{k}, k=\right.$ $1, \ldots, p\}$ denotes the unitary basis vectors of the control space $\mathcal{V}$. However, such a computation is huge for space of large dimension, since it requires $p$ integrations of the evolution model for each required value of the gradient functional. Adjoint models as introduced in optimal control theory by J.L. Lions $[35,36]$ and seminally applied in meteorology in [32] will allow us to compute the gradient functional in a single integration. To obtain the adjoint equation, the first equation of model (3) is multiplied by an adjoint variable $\lambda \in \mathcal{W}\left(t_{0}, t_{f}\right)$. The result is then integrated on $\left[t_{0}, t_{f}\right]$ :

$$
\begin{aligned}
& \int_{t_{0}}^{t_{f}}\left\langle\partial_{t} d X(t), \lambda(t)\right\rangle_{\mathcal{V}} d t \\
& \quad+\int_{t_{0}}^{t_{f}}\left\langle\left(\partial_{X} \mathbb{M}\right) d X(t), \lambda(t)\right\rangle_{\mathcal{V}} d t=0
\end{aligned}
$$

After an integration by parts of the first term and using the second equation of the differential model (3), we finally get:

$$
\begin{gathered}
-\int_{t_{0}}^{t_{f}}\left\langle-\partial_{t} \lambda(t)+\left(\partial_{X} \mathbb{M}\right)^{*} \lambda(t), d X(t)\right\rangle_{\mathcal{V}} d t \\
=\left\langle\lambda\left(t_{f}\right), d X\left(t_{f}\right)\right\rangle_{\mathcal{V}}-\left\langle\lambda\left(t_{0}\right), \delta \eta\right\rangle_{\mathcal{V}}
\end{gathered}
$$

where the adjoint operator $\left(\partial_{X} \mathbb{M}\right) *$ is defined by the scalar product:

$\forall x \in \mathcal{V}, \forall y \in \mathcal{V} \quad\left\langle\left(\partial_{X} \mathbb{M}\right) x, y\right\rangle_{\mathcal{V}}=\left\langle x,\left(\partial_{X} \mathbb{M}\right)^{*} y\right\rangle_{\mathcal{V}}$

In order to obtain an accessible expression for the cost function gradient, the adjoint variable is defined as a solution of the following adjoint problem:

Given $\eta \in \mathcal{V}, t_{f}>t_{0}$ and $X(t)$ solution of (1),

let us define $\lambda \in \mathcal{W}\left(t_{0}, t_{f}\right)$ such that:

$\left\{\begin{array}{l}-\partial_{t} \lambda(t)+\left(\partial_{X} \mathbb{M}\right)^{*} \lambda(t)=\left(\partial_{X} \mathbb{H}\right)^{*} R^{-1}(Y-\mathbb{H}(X(t))) \\ \quad \forall t \in] t_{0}, t_{f}[ \\ \lambda\left(t_{f}\right)=0\end{array}\right.$

In the same way as for the direct model, we assume that given $\eta \in \mathcal{V}, t_{f}>t_{0}$ and $X \in \mathcal{W}\left(t_{0}, t_{f}\right)$ solution of problem (1), there exists a unique function $\lambda \in \mathcal{W}\left(t_{0}, t_{f}\right)$ solution of problem (9). We also assume that this solution depends continuously on $\eta$ (i.e: $\mathcal{V} \rightarrow \mathcal{V}: \eta \mapsto \lambda(\eta, t)$ is continuous $\forall t \in] t_{0}, t_{f}[$ ).

Functional Gradient Replacing in equation (5) the second member of (9) and using (7) with the final condition of (9), we obtain:

$\left\langle\frac{\partial J}{\partial \eta}, \delta \eta\right\rangle_{\mathcal{V}}=-\left\langle\lambda\left(t_{0}\right), \delta \eta\right\rangle_{\mathcal{V}}+\left\langle B^{-1}\left(X\left(t_{0}\right)-X_{0}\right), \delta \eta\right\rangle_{\mathcal{V}}$

The cost function derivative with respect to $\eta$ finally reads:

$\frac{\partial J}{\partial \eta}=-\lambda\left(t_{0}\right)+B^{-1}\left(X\left(t_{0}\right)-X_{0}\right)$.

As a consequence, given a solution $X(t)$ of the direct model (1), the functional gradient can be computed with a backward integration of the adjoint model (9). The adjoint variable then enables to update the initial condition, by canceling the gradient defined in (10):

$X\left(t_{0}\right)=X_{0}+B \lambda\left(t_{0}\right)$

where $B$ is the pseudo inverse of $B^{-1}$ [6]. As the complexity of the adjoint model integration is similar to the integration of the direct model, the use of this technique appears to be very efficient for state space of large dimension. A synoptic of the overall technique is given in Algorithm 1.

Algorithm 1 Perfect Model

Let $X\left(t_{0}\right)=X_{0}$.

(i) From $X\left(t_{0}\right)$, compute $\left.X(t), \forall t \in\right] t_{0}, t_{f}$ [ with a forward integration of system (1).

(ii) With $X(t)$, realize a backward integration of the adjoint variable with the system (9).

(iii) Update the initial condition $X\left(t_{0}\right)$ with relation (11).

(iv) Return to (i) and repeat until a convergence criterion.

This first approach is widely used in environmental sciences for the analysis of geophysical flows [32, 57]. For these applications, the involved dynamical model are assumed to reflect faithfully the evolution of the observed phenomenon. 
However, such modeling seems to us irrelevant in image analysis since the different models on which we can rely on are usually inaccurate due for instance to 3D-2D projections, varying lighting conditions, or completely unknown boundary conditions. Considering imperfect dynamical models is now related to an optimization problem where the control variable is related to the whole trajectory of the state variable. This is the kind of problem we are facing in this paper.

\subsection{Data Assimilation with Imperfect Model}

The dynamical model we now consider is defined up to a control function $v \in \mathcal{W}\left(t_{0}, t_{f}, \mathcal{V}\right)$, where $v(t) \in \mathcal{V}$. As previously, the initial state is defined up to the control variable $\eta \in \mathcal{V}$. We are now facing an imperfect dynamical system which depends on the whole trajectory of the model control function and on the initial state control variable. Formally, the system associated to an imperfect model reads:

Given $(\nu, \eta) \in(\mathcal{W}, \mathcal{V})$

let us define $X \in \mathcal{W}\left(t_{0}, t_{f}\right)$ such that

$\left\{\begin{array}{l}\left.\partial_{t} X(t)+\mathbb{M}(X(t), t)=v(t) \quad \forall t \in\right] t_{0}, t_{f}[, \\ X\left(t_{0}\right)=X_{0}+\eta\end{array}\right.$

Cost Function As before, the objective function $J: \mathcal{W} \times$ $\mathcal{V} \rightarrow \mathbb{R}$ gathers a measurement discrepancy term and penalization terms on the control variables norms:

$$
\begin{aligned}
J(v, \eta)= & \frac{1}{2} \int_{t_{0}}^{t_{f}}\|Y-\mathbb{H}(X(v(t), \eta, t))\|_{R}^{2} d t+\frac{1}{2}\|\eta\|_{B}^{2} \\
& +\frac{1}{2} \int_{t_{0}}^{t_{f}}\|v(t)\|_{Q}^{2} d t .
\end{aligned}
$$

The norm $\|\cdot\|_{Q}$ is associated to the scalar product $\left\langle Q^{-1}, \cdot\right\rangle_{V}$, where $Q$ is a symmetric positive defined endomorphism of $\mathcal{V}$ called the model covariance matrix. We aim here at finding a minimizer $(\eta, v)$ of the cost function that is of least energy and that minimizes along time the deviations between the available measurements and the state variable.

Differential Model In the aim of computing partial derivatives of the cost function with respect to the control variables, system (12) is first differentiated with respect to $(\nu, \eta)$ in the direction $(\delta v, \delta \eta)$ :

Given $(\nu, \eta) \in(\mathcal{W}, \mathcal{V}), X(t)$ solution of (12) and a perturbation $(\delta \nu, \delta \eta) \in(\mathcal{W} \times \mathcal{V})$,

$d X=\frac{\partial X}{\partial v} \delta v+\frac{\partial X}{\partial \eta} \delta \eta \in \mathcal{W}\left(t_{0}, t_{f}\right)$, is such that:

$$
\left\{\begin{array}{l}
\left.\partial_{t} d X(t)+\left(\partial_{X} \mathbb{M}\right) d X(t)=\delta v(t) \quad \forall t \in\right] t_{0}, t_{f}[, \\
d X\left(t_{0}\right)=\delta \eta
\end{array}\right.
$$

The differentiation of cost function (13) with respect to $\eta$ has been previously computed and given in equation (5). The differentiation with respect to $v$ in the direction $\delta v$ reads:

$$
\begin{aligned}
\left\langle\frac{\partial J}{\partial v}, \delta v\right\rangle_{\mathcal{W}} \\
=-\int_{t_{0}}^{t_{f}}\left\langle Y-\mathbb{H}(X),\left(\partial_{X} \mathbb{H}\right) \frac{\partial X}{\partial v} \delta v(t)\right\rangle_{R} d t \\
\quad+\int_{t_{0}}^{t_{f}}\left\langle\partial_{t} X(t)+\mathbb{M}(X(t)), \delta v(t)\right\rangle_{Q} d t \\
=-\int_{t_{0}}^{t_{f}}\left\langle\left(\partial_{X} \mathbb{H}\right)^{*} R^{-1}(Y-\mathbb{H}(X)), \frac{\partial X}{\partial v} \delta v(t)\right\rangle_{\mathcal{V}} d t \\
\quad+\int_{t_{0}}^{t_{f}}\left\langle Q^{-1}\left(\partial_{t} X(t)+\mathbb{M}(X(t)), \delta v(t)\right\rangle_{\mathcal{V}} d t .\right.
\end{aligned}
$$

Adjoint Evolution Model Similarly to the previous case, the first equation of model (14) is multiplied by an adjoint variable $\lambda \in \mathcal{W}\left(t_{0}, t_{f}\right)$ and integrated on $\left[t_{0}, t_{f}\right]$

$$
\begin{aligned}
\int_{t_{0}}^{t_{f}} & \left\langle\partial_{t} d X(t), \lambda(t)\right\rangle_{\mathcal{V}} d t \\
& \quad+\int_{t_{0}}^{t_{f}}\left\langle\partial_{X} \mathbb{M}(X(t)) d X(t), \lambda(t)\right\rangle_{\mathcal{V}} d t \\
= & \int_{t_{0}}^{t_{f}}\langle\delta v(t), \lambda(t)\rangle_{\mathcal{V}} d t .
\end{aligned}
$$

After an integration by parts of the first term and using the second equation of the differential model (14), we finally get:

$$
\begin{gathered}
-\int_{t_{0}}^{t_{f}}\left\langle-\partial_{t} \lambda(t)+\left(\partial_{X} \mathbb{M}\right)^{*} \lambda(t), d X(t)\right\rangle_{\mathcal{V}} d t \\
=\left\langle\lambda\left(t_{f}\right), d X\left(t_{f}\right)\right\rangle_{\mathcal{V}}-\left\langle\lambda\left(t_{0}\right), \delta \eta\right\rangle_{\mathcal{V}} \\
\quad-\int_{t_{0}}^{t_{f}}\langle\lambda(t), \delta \nu(t)\rangle_{\mathcal{V}} d t
\end{gathered}
$$

As previously, in order to exhibit an expression of the gradient of the cost function from the adjoint variable, we define the following adjoint problem:

Given $(v, \eta) \in(\mathcal{W}, \mathcal{V}), t_{f}>t_{0}$ and $X(t)$ a solution of (12),

we define $\lambda \in \mathcal{W}\left(t_{0}, t_{f}\right)$, such that:

$$
\left\{\begin{array}{l}
-\partial_{t} \lambda(t)+\left(\partial_{X} \mathbb{M}\right)^{*} \lambda(t)=\left(\partial_{X} \mathbb{H}\right)^{*} R^{-1}(Y-\mathbb{H}(X(t))) \\
\quad \forall t \in] t_{0}, t_{f}[ \\
\lambda\left(t_{f}\right)=0 .
\end{array}\right.
$$

Functional Gradient Combining equations (5) and (15), the functional gradient is given by:

$$
\left\langle\frac{\partial J}{\partial v}, \delta v\right\rangle_{\mathcal{W}}+\left\langle\frac{\partial J}{\partial \eta}, \delta \eta\right\rangle_{\mathcal{V}}
$$




$$
\begin{gathered}
=\int_{t_{0}}^{t_{f}}\left\langle Q^{-1}\left(\partial_{t} X(t)+\mathbb{M}(X(t)), \delta v(t)\right\rangle \mathcal{V} d t\right. \\
+\left\langle B^{-1}\left(X\left(t_{0}\right)-X_{0}\right), \delta \eta\right\rangle_{\mathcal{V}} \\
-\int_{t_{0}}^{t_{f}}\left\langle\left(\partial_{X} \mathbb{H}\right)^{*} R^{-1}(Y-\mathbb{H}(X(t))),\right. \\
\underbrace{\frac{\partial X}{\partial w} \delta v(t)+\frac{\partial X}{\partial \eta} \delta \eta}_{d X(t)}\rangle_{\mathcal{V}} d t
\end{gathered}
$$

Introducing (16) and (17), we obtain:

$$
\begin{aligned}
\left\langle\frac{\partial J}{\partial v}, \delta \nu\right\rangle_{\mathcal{W}}+\left\langle\frac{\partial J}{\partial \eta}, \delta \eta\right\rangle_{\mathcal{V}} \\
=\int_{t_{0}}^{t_{f}}\left\langle Q^{-1}\left(\partial_{t} X(t)+\mathbb{M}(X(t))-\lambda(t), \delta v(t)\right\rangle_{\mathcal{V}} d t\right. \\
\quad-\left\langle\lambda\left(t_{0}\right), \delta \eta\right\rangle_{\mathcal{V}}+\left\langle B^{-1}\left(X\left(t_{0}\right)-X_{0}\right), \delta \eta\right\rangle_{\mathcal{V}} \\
=\left\langle Q^{-1}\left(\partial_{t} X+\mathbb{M}(X)-\lambda, \delta v\right\rangle_{\mathcal{W}}\right. \\
\quad+\left\langle-\lambda\left(t_{0}\right)+B^{-1}\left(X\left(t_{0}\right)-X_{0}\right), \delta \eta\right\rangle_{\mathcal{V}}
\end{aligned}
$$

The derivatives of the cost function with respect to $v$ and $\eta$ are identified as:

$$
\begin{aligned}
& \frac{\partial J}{\partial v}=Q^{-1}\left(\partial_{t} X+\mathbb{M}(X)\right)-\lambda, \\
& \frac{\partial J}{\partial \eta}=-\lambda\left(t_{0}\right)+B^{-1}\left(X\left(t_{0}\right)-X_{0}\right) .
\end{aligned}
$$

Canceling these components and introducing $Q$ and $B$, the respective pseudo inverses of $Q^{-1}$ and $B^{-1}$, we get:

$$
\left\{\begin{array}{l}
\partial_{t} X(t)+\mathbb{M}(X(t))=Q \lambda(t), \\
X\left(t_{0}\right)-X_{0}=B \lambda\left(t_{0}\right) .
\end{array}\right.
$$

The second equation still constitutes an incremental update of the initial condition. However, the integration of this system requires the knowledge of the whole adjoint variable trajectory, which itself depends on the state variable through (17). This system can be in practice integrated introducing an incremental splitting strategy.

\section{Incremental Function Defining the state variable as}

$$
\left\{\begin{array}{l}
X(t)=\tilde{X}(t)+d f(t) \quad \forall t \in\left[t_{0}, t_{f}\right], \\
\tilde{X}\left(t_{0}\right)=X_{0}
\end{array}\right.
$$

where $\tilde{X}(t)$ is either a fixed component or a previously estimated trajectory of the state variable, equation (20) can be separated and written as:

$$
\begin{aligned}
& \left.\partial_{t} \tilde{X}(t)+\mathbb{M}(\tilde{X}(t))=0 \quad \forall t \in\right] t_{0}, t_{f}[, \\
& \left.\partial_{t} d f(t)+\partial_{\tilde{X}} \mathbb{M}(\tilde{X}(t)) d f(t)=Q \lambda(t) \quad \forall t \in\right] t_{0}, t_{f}[.
\end{aligned}
$$

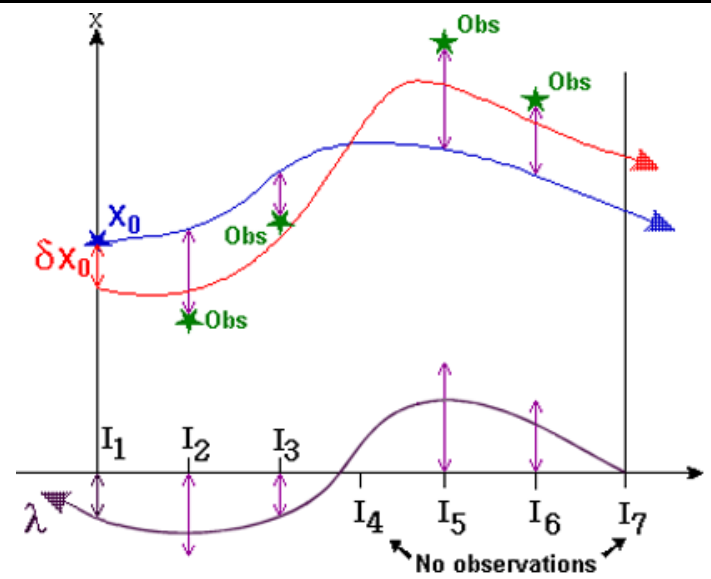

Fig. 1 Assimilation algorithm principle. This figure gives a synoptic of the overall principle of the method. After an integration of the initial condition $X_{0}$, a backward integration of the adjoint variable relying on a measurement discrepancy enables to compute a forward incremental correction trajectory and so on...

As a consequence, the update of the state variable $X$ is driven by an incremental function $d f$. The adjoint variable $\lambda$ is obtained from a backward integration of (17) and from the trajectory of $\tilde{X}$. The initial value of this incremental function is then obtained from (19) and reads:

$d f\left(t_{0}\right)=B \lambda\left(t_{0}\right)$

Equations (12), (21), (22), (23) and (24) give rise to a variational assimilation method with imperfect dynamical model. A sketch of the whole process is summarized in Algorithm 2.

Algorithm 2 Let $X\left(t_{0}\right)=X_{0}$.

(i) From $X\left(t_{0}\right)$, compute $\left.X(t), \forall t \in\right] t_{0}, t_{f}$ [ with a forward integration of system (22).

(ii) $X(t)$ being given, realize a backward integration of the adjoint variable with the system (17).

(iii) Compute $d f\left(t_{0}\right)$, the initial value of the incremental function with equation (24).

(iv) From $d f\left(t_{0}\right)$, compute $\left.d f(t), \forall t \in\right] t_{0}, t_{f}$ [ with a forward integration of system (23).

(v) Update $X=X+d f$.

(vi) Return to (ii) and repeat until convergence.

The algorithm principles are also schematically pictured on Fig. 1.

\subsection{Additional Ingredients}

Before turning to the application of such a framework, let us note that the system we have presented so far can be slightly modified and enriched considering a final condition or additional types of observations. 
Final Condition Symmetrically to the initial condition equation, a final target state can eventually be added through an additional equation:

Given $(\nu, \eta, w) \in(\mathcal{W}, \mathcal{V}, \mathcal{V})$, let us

define $X \in \mathcal{W}\left(t_{0}, t_{f}\right)$, such that:

$\left\{\begin{array}{l}\left.\partial_{t} X(t)+\mathbb{M}(X(t), t)=v(t) \quad \forall t \in\right] t_{0}, t_{f}[, \\ X\left(t_{0}\right)=X_{0}+\eta, \\ X\left(t_{f}\right)=X_{f}+w,\end{array}\right.$

with $w \in \mathcal{V}$ an additional control variable. The cost function to minimize in this case incorporates an extra penalization term on the norm of this new control variable:

$$
\begin{aligned}
J(v, \eta, w)= & \frac{1}{2} \int_{t_{0}}^{t_{f}}\|Y-\mathbb{H}(X(v(t), \eta, w, t))\|_{R}^{2} d t \\
& +\frac{1}{2}\|\eta\|_{B}^{2}+\frac{1}{2} \int_{t_{0}}^{t_{f}}\|v(t)\|_{Q}^{2} d t+\frac{1}{2}\|w\|_{F}^{2} .
\end{aligned}
$$

The norm $\|\cdot\|_{F}$ is associated to the scalar product $\left\langle F^{-1} \cdot, \cdot\right\rangle_{I}$. The differentiation of the cost function (26) with respect to $w$ in the direction $\delta w$ is:

$$
\begin{aligned}
\left\langle\frac{\partial J}{\partial w}, \delta w\right\rangle_{\mathcal{V}} & \\
= & -\int_{t_{0}}^{t_{f}}\left\langle\left(\partial_{X} \mathbb{H}\right)^{*} R^{-1}(Y-\mathbb{H}(X)), \frac{\partial X}{\partial w} \delta w\right\rangle_{\mathcal{V}} d t \\
& +\left\langle F^{-1}\left(X\left(t_{f}\right)-X_{f}\right), \delta w\right\rangle_{\mathcal{V}} .
\end{aligned}
$$

The introduction of an additional equation on the final state modifies the final condition of the adjoint model. It now reads:

Given $(\nu, \eta, w) \in(\mathcal{W}, \mathcal{V}, \mathcal{V}), t_{f}>t_{0}$ and

$X(t)$ a solution of (25), let the adjoint variable

$\lambda \in \mathcal{W}\left(t_{0}, t_{f}\right)$, be defined such that:

$\left\{\begin{array}{l}-\partial_{t} \lambda(t)+\left(\partial_{X} \mathbb{M}\right)^{*} \lambda(t)=\left(\partial_{X} \mathbb{H}\right)^{*} R^{-1}(Y-\mathbb{H}(X)) \\ \quad \forall t \in] t_{0}, t_{f}[ \\ \lambda\left(t_{f}\right)=F^{-1}\left(X_{f}-X\left(t_{f}\right)\right) .\end{array}\right.$

Several Observations If we consider a set of observations $Y_{i} \in \mathcal{O}_{i}, i \in\{1, \ldots, N\}$ related to the state variable through the observation operators $\mathbb{H}_{i}\left(V, \mathcal{O}_{i}\right)$, the measurement discrepancy term of the cost function becomes:

$\frac{1}{2} \sum_{i=1}^{N}\left\|Y_{i}-\mathbb{H}_{i}(X, \eta, t)\right\|_{R_{i}}^{2}$.
The norms $\|\cdot\|_{R_{i}}$ involved in this term are associated to the scalar products $\left\langle R_{i}{ }^{-1}, \cdot\right\rangle_{\mathcal{O}_{i}}$. Such a term also implies a straightforward modification of the adjoint model:

Given $\eta \in \mathcal{V}, t_{f}>t_{0}$ and $X(t)$ solution of (1),

let the adjoint $\lambda \in \mathcal{W}\left(t_{0}, t_{f}\right)$, be such that:

$$
\left\{\begin{array}{l}
-\partial_{t} \lambda(t)+\left(\partial_{X} \mathbb{M}\right)^{*} \lambda(t) \\
\left.\quad=\sum_{i=1}^{N}\left(\partial_{X} \mathbb{H}_{i}\right)^{*} R_{i}^{-1}\left(Y_{i}-\mathbb{H}_{i}(X)\right) \quad \forall t \in\right] t_{0}, t_{f}[ \\
\lambda\left(t_{f}\right)=0 .
\end{array}\right.
$$

\subsection{Discussion on Convergence}

The convergence criterion introduced in Algorithm 1 can be defined from the norm of the objective function gradient, namely $\left|\lambda\left(t_{0}\right)\right|<\alpha$, where $\alpha$ is a given threshold. The value of this threshold must be as small as possible, but above numerical errors due to the discretization schemes used. An empirical test related to the numerical stability of the adjoint operators discretization [16, 23] enables to determine this threshold value. Referring to Taylor expansion, this test consists in checking that the ratio

$\lim _{\alpha \rightarrow 0} \frac{J(X+\alpha d X)-J(X)}{\alpha \nabla J} \rightarrow 1$.

The numerator is computed through finite differences, whereas the denominator is obtained by the adjoint model. The test thus enables to find, for a given set of measurements, the smallest value of $\alpha$ that respects this limit.

In order to optimize the minimization process, different types of efficient gradient descent strategies can be used (Conjugate Gradient, quasi Newton methods...). In this work, we use a simple fixed step gradient descent.

As for the global convergence of the method, the minimization problem has a unique solution if the functional $J$ is convex, lower semi-continuous and if:

$\lim _{\|\nu\|_{\mathcal{W}} \rightarrow \infty,\|\eta\|_{\mathcal{V}} \rightarrow \infty} J(\nu, \eta)=\infty$.

If the involved models $\mathbb{M}$ and $\mathbb{H}$ are nonlinear, the functional will be likely not convex. In this case, if the minimization strategy is adapted [58], the process will only converge to a local minimum which depends on the chosen initialization. Hence, the initialization term is, in general, of great importance to obtain a good solution. Nevertheless, let us remind that the control parameter on the initial condition equation enables to model an incertitude on this initial state.

\subsection{Relations with the Kalman Filter and the Kalman Smoother}

We briefly discuss in this section the relations between the previous system and the Kalman filter and smoother. As 
variational assimilation technique and Kalman filtering are based on very same kind of systems, both techniques share some similarities. Theoretical equivalence between the two techniques have been proved for linear systems (both model and observation operators are linear) [8,34]. One of the main differences concerns the goal sought by each technique. Kalman filter aims at computing recursively in time the two first moments of the conditional distribution of the state variables given all the available observations [2]formally the sequence of past observations in case of filtering and the complete sequence in case of smoothingwhereas a variational assimilation technique aims at estimating the minimal cost trajectory in batch mode. In case of a linear Gaussian system, the first moment of the filtering distribution and the solution of the variational technique are equivalent. This is not the case for nonlinear systems. Furthermore, unlike Kalman filtering, variational approaches do not provide any covariance of the estimation error. From a practical and computational point of view, there exists also some differences between both techniques. Kalman filtering requires at each iteration to inverse the estimation error covariance matrix in order to compute the so called Kalman gain matrix. The dimension of this matrix is the square of the size of the state vector. As a consequence, in applications involving large state vectors, the computational cost of Kalman filtering is completely unaffordable without simplifications. At the opposite, variational assimilation does not require such an inversion. The estimation is here done iteratively by forward-backward integrations and not directly through a recursive process like in Kalman filtering. This point makes variational assimilation methods very attractive for the tracking of high dimensional features.

\subsection{Batch Filtering vs. Recursive Tracking}

As already mentioned in previous section, one of the main differences between Bayesian filters and variational assimilation techniques relies on the underlying integration that is operated. The forward recursive expression of the former makes Bayesian filter well suited to real time tracking. They are on the other side only efficient for low dimensional state spaces. The latter techniques are inherently batch processes as they are based on forward-backward integration schemes. Unless relying on temporal sliding windows such a characteristic makes their use difficult for real time tracking. Nevertheless, their abilities to cope with large dimensional state spaces and with (highly) nonlinear differential dynamics make them interesting for a batch video processing. Such analysis is interesting when one wishes to extract a continuous and temporal coherent sequence of features with respect to a specified dynamic from a sequence of noisy and incomplete data. As we will show in the following sections this is of particular interest when one aims at analyzing fluid flows from image sequences. We also believe that such a scheme could bring valuable information for the analysis of deformations in medical images or in the domain of video processing for purposes of video inpainting, object recolorization, morphing or video restoration. We give some hints about these potential applications in the following.

\section{Application to Curve Tracking}

Before dealing with the most complex issue of jointly tracking curve and motion, we will first focus in this section on the application of the variational data assimilation to curve tracking from an image sequence. In this first application, the motion fields driving the curve will be considered as reliable external inputs of the system. For such a system, different types of measurements will be explored.

\subsection{Contour Representation and Evolution Law}

As we wish to focus on the tracking of nonparametric closed curves that may exhibit topology changes during the time of the analyzed image sequence, we will rely on an implicit level set representation of the curve of interest $\Gamma(t)$ at time $t \in\left[t_{0}, t_{f}\right]$ of the image sequence $[45,54]$. Within that framework, the curve $\Gamma(t)$ enclosing the target to track is implicitly described by the zero level set of a function $\phi(\boldsymbol{x}, t): \Omega \times \mathbb{R}+\rightarrow \mathbb{R}:$

$\Gamma(t)=\{\boldsymbol{x} \in \Omega \mid \phi(\boldsymbol{x}, t)=0\}$,

where $\Omega$ stands for the image spatial domain. This representation enables an Eulerian representation of the evolving contours. As such, it enables to avoid the ad-hoc regridding processes of the different control points required in any explicit Lagrangian - spline based - curve description. The problem we want to face consists in estimating for a whole time range the state of an unknown curve, and hence of its associated implicit surface $\phi$. To that end, we first define an a priori evolution law of the unknown surface. We will assume that the curve is transported at each frame instant by a velocity field, $\boldsymbol{w}(\boldsymbol{x}, t)=[u(\boldsymbol{x}, t), v(\boldsymbol{x}, t)]^{T}$, and diffuses according to a mean curvature motion. We will assume that this evolution law is verified only up to an additive control function. In term of implicit surface, the overall evolution law reads:

$\partial_{t} \phi+\nabla \phi \cdot \boldsymbol{w}-\varepsilon \kappa\|\nabla \phi\|=v$,

where $\kappa$ denotes the curve curvature, and $v(\boldsymbol{x}, t)$ the control function. Introducing the surface normal, equation (31) can be written as:

$\partial_{t} \phi+\underbrace{(\boldsymbol{w} \cdot \boldsymbol{n}-\varepsilon \kappa)\|\boldsymbol{\nabla} \boldsymbol{\phi}\|}_{\triangleq \mathbb{M}(\phi)}=v$, 
where the curvature and the normal are directly given in term of surface gradient:

$\kappa=\operatorname{div}\left(\frac{\nabla \phi}{\|\nabla \phi\|}\right) \quad$ and $\quad \boldsymbol{n}=\frac{\nabla \phi}{\|\nabla \phi\|}$.

As previously indicated, the motion field transporting the curve will be first assumed to be given by an external estimator. In practice, we used an efficient and robust version of the Horn and Schunck optical-flow estimator [39]. Concerning the covariance $Q$ of the control term involved in the evolution law, we fixed it to a constant diagonal matrix since it is rather difficult to infer precise model's errors. Two different ranges of value are used. For sequences where accurate motion fields can be recovered, we fix this value to a low value (typically 0.005 ). At the opposite, for sequences for which only motion fields of bad quality are obtained, this parameter is set to a higher value (0.5) as a rough dynamic is faced.

Tangent Linear Evolution Operator To apply the variational data assimilation principles, we must first define the expression of the Gâteaux derivative of the operators involved. Using equation (32), the evolution operator reads in its complete form as:

$\mathbb{M}(\phi)=\nabla \phi \cdot w-\varepsilon\|\nabla \phi\| \operatorname{div}\left(\frac{\nabla \phi}{\|\nabla \phi\|}\right)$.

This operator can be turned into a more tractable expression for our purpose:

$\mathbb{M}(\phi)=\nabla \phi^{T} \boldsymbol{w}-\varepsilon\left(\Delta \phi-\frac{\nabla^{T} \phi H(\phi) \nabla \phi}{\|\nabla \phi\|^{2}}\right)$,

where $H(\phi)$ denotes the Hessian matrix gathering the second order derivatives of $p h i$. The corresponding tangent linear operator at point function $\phi$ finally reads:

$$
\begin{aligned}
\partial_{\phi} \mathbb{M} \delta \phi= & \nabla \delta \phi^{T} \boldsymbol{w}-\varepsilon\left[\Delta \delta \phi-\frac{\nabla \phi^{T} H(\delta \phi) \nabla \phi}{\|\nabla \phi\|^{2}}\right. \\
& \left.+2 \frac{\nabla \phi^{T} H(\phi)}{\|\nabla \phi\|^{2}}\left(\frac{\nabla \phi \nabla \phi^{T}}{\|\nabla \phi\|^{2}}-I d\right) \nabla \delta \phi\right] .
\end{aligned}
$$

Operator Discretization Before going any further, let us describe the discretization schemes we considered for the evolution law. This concerns the evolution operator, the associated tangent linear operator and the adjoint evolution operator. We will denote by $\phi_{i, j}^{t}$ the value of $\phi$ at image grid point $(i, j)$ at time $t \in\left[t_{0} ; t_{f}\right]$. Using (31) and a semiimplicit discretization scheme, the following discrete evolution model is obtained:

$$
\frac{\phi_{i, j}^{t+\Delta t}-\phi_{i, j}^{t}}{\Delta t}+\mathbb{M}_{\phi_{i, j}^{t}} \phi_{i, j}^{t+\Delta t}=0 .
$$

Considering $\phi_{x}$ and $\phi_{y}$, the horizontal and vertical gradient matrices of $\phi$, the discrete operator $\mathbb{M}$ of equation (33) can be written as:

$$
\begin{aligned}
\mathbb{M}_{\phi_{i, j}^{t}} \phi_{i, j}^{t+\Delta t} & \\
= & \left(\begin{array}{c}
\left(\phi_{x}^{t+\Delta t}\right)_{i, j} \\
\left(\phi_{y}^{t+\Delta t}\right)_{i, j}
\end{array}\right)^{T} w \\
& -\frac{\varepsilon}{\left\|\nabla \phi_{i, j}^{t}\right\|^{2}}\left(\begin{array}{c}
-\left(\phi_{y}^{t}\right)_{i, j} \\
\left(\phi_{x}^{t}\right)_{i, j}
\end{array}\right)^{T} H\left(\phi_{i, j}^{t+\Delta t}\right)\left(\begin{array}{c}
-\left(\phi_{y}^{t}\right)_{i, j} \\
\left(\phi_{x}^{t}\right)_{i, j}
\end{array}\right),
\end{aligned}
$$

where we used usual finite differences for the Hessian matrix $H(\phi)$ and a first order convex scheme [54] for the advective term $\nabla \phi^{T} \boldsymbol{w}$. This scheme enables to compute the surface gradients in the appropriate direction:

$$
\begin{aligned}
\nabla \phi_{i, j}^{T} \boldsymbol{w}_{i, j}= & \max \left(u_{i, j}, 0\right)\left(\phi_{i, j}\right)_{x}^{-}+\min \left(u_{i, j}, 0\right)\left(\phi_{i, j}\right)_{x}^{+} \\
& +\max \left(v_{i, j}, 0\right)\left(\phi_{i, j}\right)_{y}^{-}+\min \left(v_{i, j}, 0\right)\left(\phi_{i, j}\right)_{y}^{+},
\end{aligned}
$$

where $(\phi)_{x}^{-}$and $(\phi)_{y}^{-}$are the left semi-finite differences, whereas $(\phi)_{x}^{+}$and $(\phi)_{y}^{+}$are the right ones. The discrete linear tangent operator introduced in (34) is similarly defined as:

$\partial_{\phi_{i, j}^{t}} \mathbb{M} \delta \phi_{i, j}^{t+\Delta t}=\mathbb{M}_{\phi_{i, j}^{t}} \delta \phi_{i, j}^{t+\Delta t}-\frac{2 \varepsilon(A B)}{\|\nabla \phi\|^{4}}\left(\begin{array}{c}\left(\delta \phi_{x}^{t+\Delta t}\right)_{i, j} \\ \left(\delta \phi_{y}^{t+\Delta t}\right)_{i, j}\end{array}\right)$,

where $A$ and $B$ are:

$$
\begin{aligned}
& A=\phi_{x}^{t} \phi_{y}^{t}\left(\phi_{x y}^{t} \phi_{x}^{t}-\phi_{x x}^{t} \phi_{y}^{t}\right)+\left(\phi_{y}^{t}\right)^{2}\left(\phi_{y y}^{t} \phi_{x}^{t}-\phi_{x y}^{t} \phi_{y}^{t}\right), \\
& B=\phi_{x}^{t} \phi_{y}^{t}\left(\phi_{x y}^{t} \phi_{y}^{t}-\phi_{y y}^{t} \phi_{x}^{t}\right)+\left(\phi_{x}^{t}\right)^{2}\left(\phi_{x x}^{t} \phi_{y}^{t}-\phi_{x y}^{t} \phi_{x}^{t}\right),
\end{aligned}
$$

and $\phi_{x x}, \phi_{x y}$ and $\phi_{y y}$ are the second order derivatives computed with central schemes. As for the iterative solver involved in the implicit discretization, we used a conjugated gradient optimization. The discretization of the adjoint evolution model is finally obtained as the transposed matrix corresponding to the discretization of the derivative of the evolution law operator.

\subsection{Initial Condition}

In order to define an initial condition, we assume that an initial contour of the target object is available. Such contours may be provided by any segmentation technique or specified by the user. Given this initial contour, we assigned a signed distance function to the implicit function at the initial time. This initial condition is also assumed to hold up to some uncertainty. More precisely, the value of $\phi\left(\boldsymbol{x}, t_{0}\right)$ is set to the distance $g\left(\boldsymbol{x}, \Gamma\left(t_{0}\right)\right)$ of the closest point of a given initial curve $\Gamma\left(t_{0}\right)$, with the convention that $g\left(\boldsymbol{x}, \Gamma\left(t_{0}\right)\right)$ is negative inside the contour, and positive outside. An additive control 
variable that models the uncertainty we have on the initial curve. This initial condition reads:

$\phi\left(\boldsymbol{x}, t_{0}\right)=g\left(\boldsymbol{x}, \Gamma\left(t_{0}\right)\right)+\eta(\boldsymbol{x})$.

The covariance matrix $B$ that models the uncertainty on the initial state has been defined as a diagonal matrix

$B(\boldsymbol{x})=I d-e^{-\left|g\left(\boldsymbol{x}, \Gamma\left(t_{0}\right)\right)\right|}$,

where $I d$ is the identity matrix. This covariance fixes a low uncertainty on the level set in the vicinity of the initial given curve. This uncertainty increases as soon as we move away from the initial contour.

\subsection{Measurement Equation}

Associated to the evolution law and to the initialization process we previously described, we have to define a measurement equation linking the unknown state variable to the available observations. In this work, the observation function $Y(t)$ is assumed to be related to the unknown state function $\phi$ through a differential operator $\mathbb{H}$. Let us recall that we are going to minimize the following functional with respect to $\eta$ and $v$ the control functions respectively associated to the initialization and to the dynamical model:

$$
\begin{aligned}
J(\eta, v)= & \int_{t_{0}}^{t_{f}}\|Y(\boldsymbol{x}, t)-\mathbb{H}(\phi(\boldsymbol{x}, t), v(t), \eta)\|_{R(t)} \\
& +\|\eta\|_{B}+\int_{t_{0}}^{t_{f}}\|v(t)\|_{Q(t)} .
\end{aligned}
$$

We here use two different measurements equation for the curve tracking issue. Let us further describe the two options we devised.

\subsubsection{Noisy Curves Observation}

The simplest measurements equation that can be settled consists in defining an observation function, $Y(t)$, in the same space as the state variable $\phi$, with the identity as measurements operator, $\mathbb{H}=I d$. Sticking first to this case, we define the observation function as the signed distance map to an observed noisy closed curve, $g(\boldsymbol{x}, \Gamma(t))$. These curves $\Gamma(t)$ are assumed to be provided by a basic threshold segmentation method or by some moving object detection method. Let us outline that such observations are generally of bad quality. As a matter of fact, thresholding may lead to very noisy curves and motion detectors are usually pruned to miss-detection or may even sometimes fail to provide any detection mask when the object motion is too low. In this case, we define the covariance $R$ of the measurements discrepancy as

$R(\boldsymbol{x}, t)=R_{\min }+\left(R_{\max }-R_{\min }\right)\left(I d-e^{-|Y(\boldsymbol{x}, t)|}\right)$.
This function has lower values in the vicinity of the observed curves $\left(R_{\min }=10\right)$ and higher values faraway from them $\left(R_{\max }=50\right)$. When there is no observed curve, all the corresponding values of this covariance function are set to infinity.

\subsubsection{Image Observation Based on Local Probability Density}

The previous observations require the use of an external segmentation process which we would like to avoid. To directly rely on the image data, a more complex measurement equation can be built to deal with local probability distributions of the intensity function. This model compares at each point of the image domain $\Omega$ a local photometric histogram to two global probability density functions $\rho_{o}$ and $\rho_{b}$ modeling respectively the object and background intensity distributions. These two distributions are assumed to be estimated from the initial location of the target object. The measurements equation we propose in this case reads:

$$
\begin{aligned}
F(\phi, I)(\boldsymbol{x}, t)= & {\left[1-d_{B}\left(\rho_{V_{x}}, \rho_{o}\right)\right]^{2} \mathbf{1}_{\phi(\boldsymbol{x})<0} } \\
& +\left[1-d_{B}\left(\rho_{V_{x}}, \rho_{b}\right)\right]^{2} \mathbf{1}_{\phi(x) \geq 0} \\
= & \epsilon(\boldsymbol{x}, t),
\end{aligned}
$$

where $d_{B}$ is the Bhattacharya probability density distance measure, $\rho_{V_{x}}$ is the probability density in the neighborhood $V_{\boldsymbol{x}}$ of a pixel $\boldsymbol{x}$ and $\epsilon$ is the function modeling the observation error. In our applications, the local neighborhood is defined as points such that $\boldsymbol{y} \in V_{\boldsymbol{x}}$ if $|\boldsymbol{y}-\boldsymbol{x}| \leq 3$. Replacing the densities with the intensity average, we retrieve the well-known Chan and Vese functional proposed for image segmentation [13]. The Bhattacharya distance is in our case defined as:

$d_{B}\left(\rho_{1}, \rho_{2}\right)=\int_{0}^{255} \sqrt{\rho_{1}(z) \rho_{2}(z)} d z$.

This distance equals one if the two densities are identical. Let us note that the discrepancy between the measurements and the state variable does not appear explicitly. The measurement operator gathers here the measurements (the local intensity distribution) and the relation between the measurements and the state variable (the appropriate Bhattacharya distance). The corresponding linear tangent operator in the sense of distributions [13] is:

$\partial_{\phi} F=\left(\left[1-d_{B}\left(\rho_{V_{x}}, \rho_{o}\right)\right]^{2}-\left[1-d_{B}\left(\rho_{V_{x}}, \rho_{b}\right)\right]^{2}\right) \delta(\phi)$,

where $\delta(\cdot)$ is the Dirac function. In this case the covariance associated to the measurements error $\epsilon$ has been fixed to a diagonal matrix corresponding to the minimal empiric local photometric covariance:

$R(\boldsymbol{x}, t)=\mathbb{E}\left[\operatorname{Min}\left(\left(1-d_{B}\left(\rho_{V_{x}}, \rho_{o}\right)\right)^{2},\left(1-d_{B}\left(\rho_{V_{x}}, \rho_{b}\right)\right)^{2}\right)\right]$. 
Fig. 2 Clouds sequence. Top row: Sample of observed curves. Bottom row: Recovered results superimposed on the corresponding image

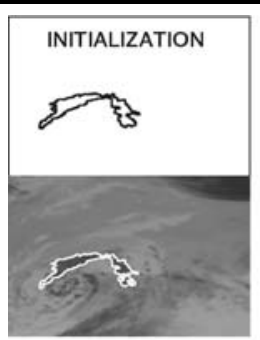

$t_{0}$

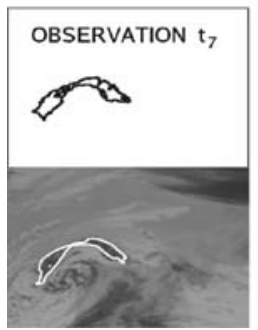

$t_{7}$

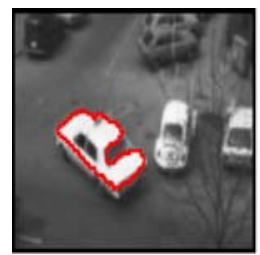

$\mathrm{t}=0$

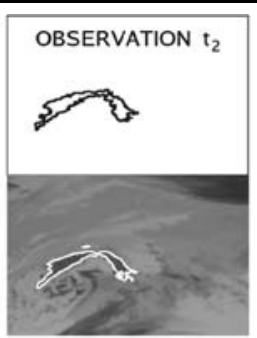

$t_{2}$

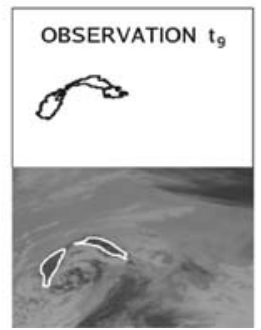

$t_{9}$

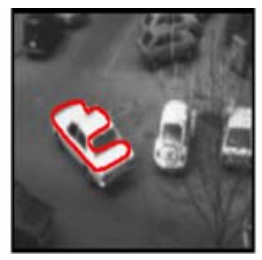

$\mathrm{t}=9$

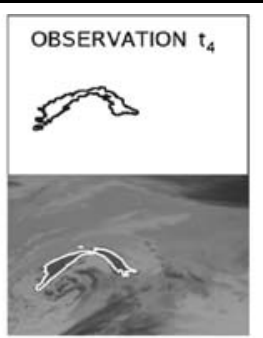

$t_{4}$

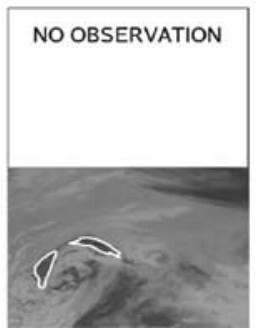

$t_{10}$

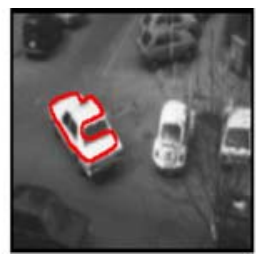

$\mathrm{t}=17$

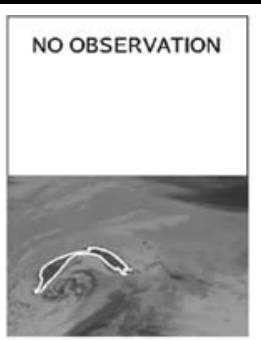

$t_{6}$

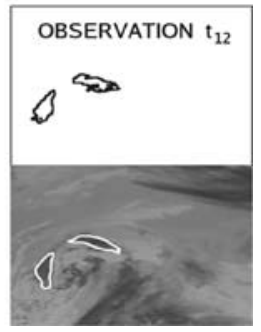

$t_{12}$

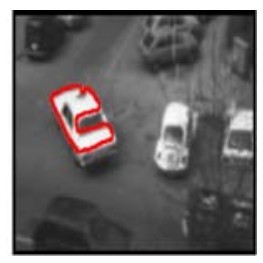

$\mathrm{t}=25$

\subsection{Experimental Results}

First of all, let us recall that for these first series of experiments the motion field transporting the curve of interest is assumed to be provided through an external motion estimation technique [39]. Moreover, we assume that an initial curve is provided either by a segmentation process in the first image or given by the user.

The first example shown on Fig. 2 concerns a meteorological image sequence of the Meteosat infra red channel. We rely here on the simplest measurement model built from noisy curves in Sect. 3.3.1. It is extracted through a simple threshold technique that selects a photometric Infra-red level line. Thus, we aim at tracking an iso-temperature curve. To demonstrate the robustness of our technique, we assume that this observation is missing at some instant of the sequence. The results and the observed curves are given in Fig. 2. The obtained results illustrate that the proposed technique conserves the adaptive topology property of level set methods, and at the same time, despite missing measurements, maintains a temporal consistency of the curves extracted.

To demonstrate the interest of the second observation model based on the local probability density, we applied our assimilation technique with such measurements for the tracking of a car in the Hamburg taxi sequence. For this example, the initial curve is still given by a thresholding technique. The results are presented on Fig. 3. They show that the proposed system enables to track accurately and in continuous way the changing shape of an object of interest.

To further illustrate the interest of the proposed technique, we also tracked a curve delineating alphabetic letters. The measurements consist of a set of four binary letters images, as shown on Fig. 4. On the same figure, we plotted the results obtained at intermediate instants. This toy example illustrates that the method provides a consistent continuous sequence of curves (with respect to a given dynamics) and how the curve moves progressively between two distinct shapes.

We then applied the technique to track a running tiger. This noisy sequence is composed of 27 frames and exhibits motion blur at some places. The measurements are provided here by local photometric histograms. The initial curve that determines probability density functions of the tiger and the background has been obtained with a simple threshold technique. The results obtained are shown on Fig. 5. For this sequence we also plotted on Fig. 6 a set of segmentation obtained through a Chan and Vese segmentation process [13] based on the same data model than our measurements model (see (36-37)) together with an additional penalization term on the curve length. As can be observed from Fig. 6 the masks obtained with this spatial segmentation technique are of good quality for some frames. Nevertheless, they appear to be unstable along time and would require a delicate tuning 
Fig. 4 Letter sequence. Result the measurement model based on the local probability densities. The curve is superimposed on the observed letter images at times $t=0,1,2,3$

Fig. 5 Tiger sequence. Result of the assimilation technique with the photometric measurement model based on the local probability densities

Fig. 6 Tiger sequence. Successive segmentations obtained through a Chan and Vese level-set techniques with a data model based on the local probability densities measurement and a Bhattacharya distance (see (36-37)) of the assimilation process with

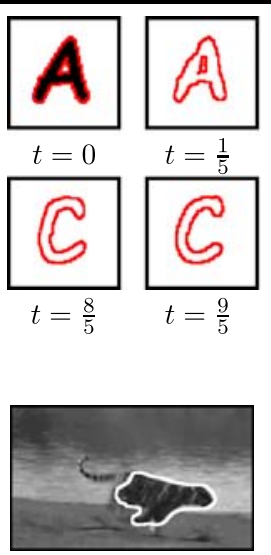

$t=0$

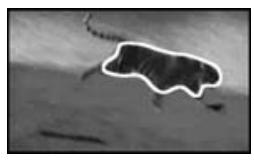

$t=10$

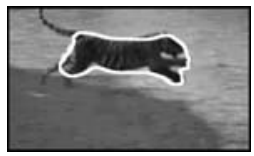

$t=20$

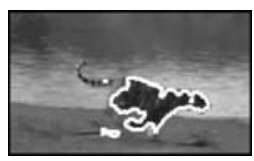

$t=0$

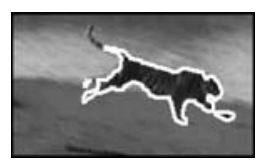

$t=9$

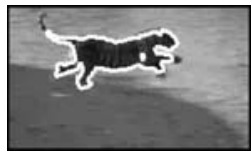

$t=19$

of the smoothing parameter at each frame to obtain a consistent sequence of curves. An optimal curvature fitting on the whole sequence would be difficult to achieve and spurious splitting or merging would hardly be avoided on some frames. At the opposite, the curves provided by the proposed technique are more stable in time and consistent with respect to the object shape and its deformations. Compared to traditional segmentation techniques the assimilation techniques provides result which reflects in a more coherent way the topology and the deformation of the target object along time. Due to the bad quality of the image sequence and to the corresponding velocity fields, we can observe that it is nevertheless difficult for the curve to fit precisely and in a continuous way to the photometric boundaries of the object.
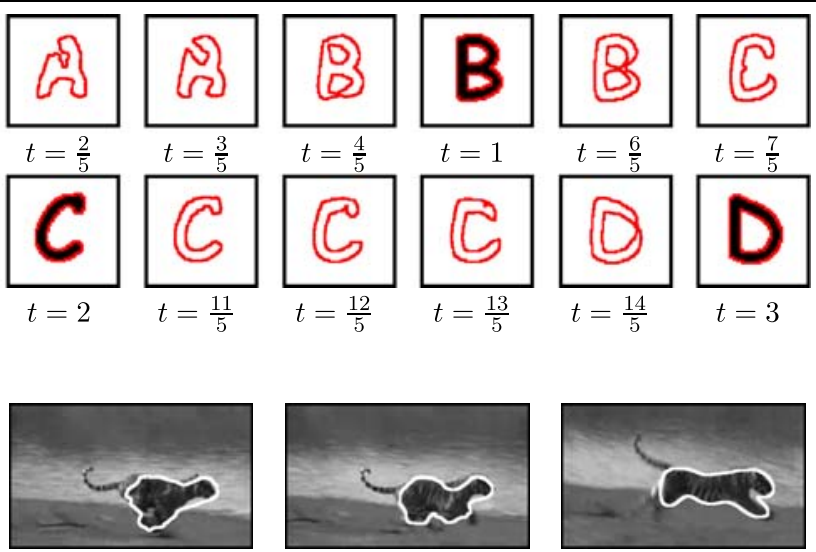

$t=2$

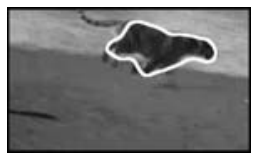

$t=12$

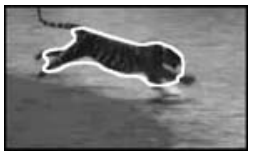

$t=22$

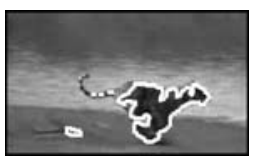

$t=2$

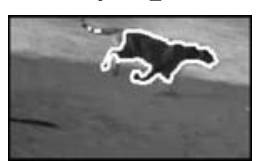

$t=12$

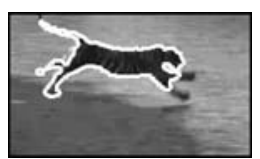

$t=21$

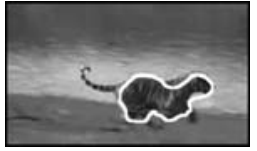

$t=4$

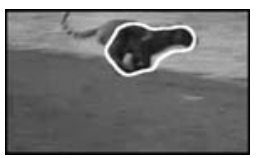

$t=14$

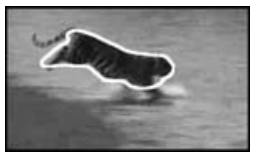

$t=24$

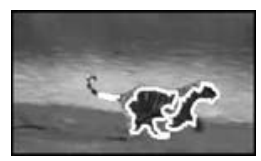

$t=4$

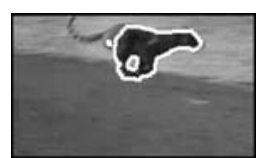

$t=14$

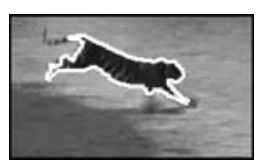

$t=23$

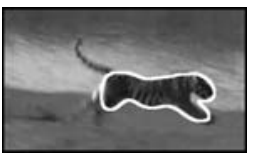

$t=7$

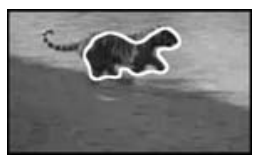

$t=17$

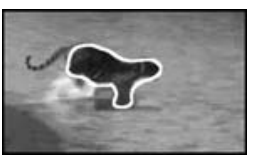

$t=26$

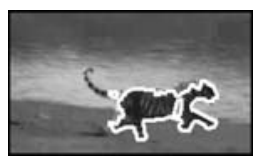

$t=5$

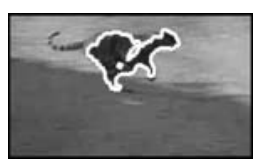

$t=16$

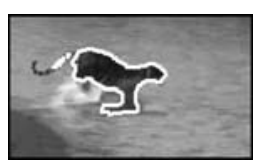

$t=26$
To further illustrate the differences between the results obtained through assimilation and successive photometric segmentations we finally run our method on a cardiac magnetic resonance imaging sequence. ${ }^{1}$ The purpose is here to track the left ventricle. The results of the method are presented on Fig. 7. As can be observed, the target region approximatively delineated in the first image by the user is well tracked: the successive deformations of the region are recovered in a coherent continuous way. The sequence of curve delineates well the evolution of a target region of interest specified at the initialization stage. In comparison, the results obtained from the same initialization with the Chan and

\footnotetext{
${ }^{1}$ http://mrel.usc.edu/class/preview.html.
} 
Fig. 7 Cardiac magnetic resonance imaging sequence. Result of the assimilation technique with the photometric measurement model based on the local probability densities. The initial target region is shown in the first image of the top row

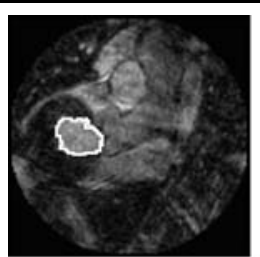

Initialization

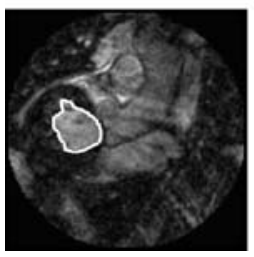

$t=4$

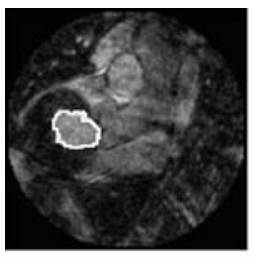

Initialization

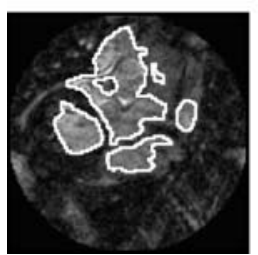

$t=4$

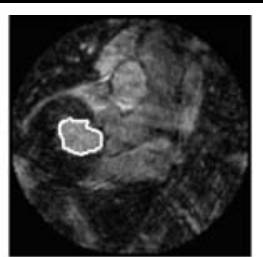

$t=1$

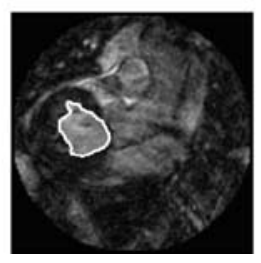

$t=5$

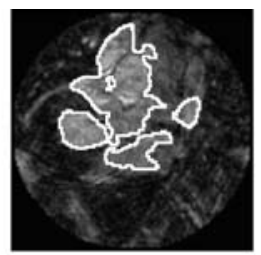

$t=1$

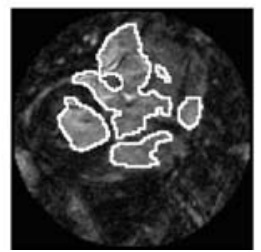

$t=5$

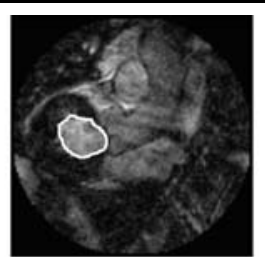

$t=2$

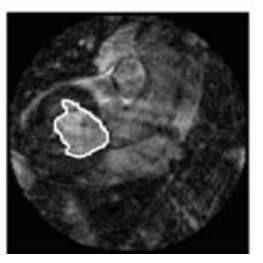

$t=6$

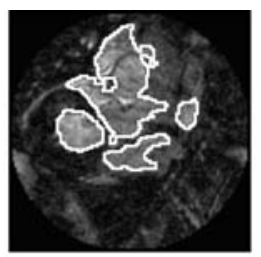

$t=2$

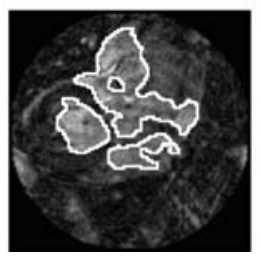

$t=6$

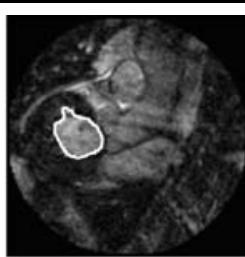

$t=3$

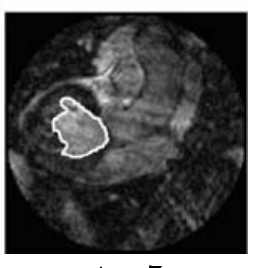

$t=7$

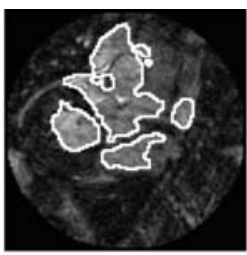

$t=3$

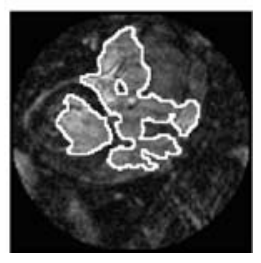

$t=7$
Vese techniques show an immediate expansion of the target region to other regions of the image characterized by the same photometric distribution (see Fig. 8). Such a process is mainly driven from frame to frame by the data term and no constraint relative to any dynamical prior of the curve is included to prevent nonplausible deformations between two frames. Incoherent merging or splitting of regions regarding the effective deformations of an object shape is maybe one of the main problems faced when running spatio-temporal analysis on the basis of consecutive single spatial analysis (even chained together through their initializations). As for the computational cost of the method, it appears to be similar to standard level set segmentation. For instance, for a 512 image sequence of 20 frames the technique takes around two minutes of computation on a standard PC.

All the results shown here have been obtained considering a sequence of velocity fields describing the motion of a region of interest. These external motion inputs have thus been assumed reliable. In complex cases involving for instance occlusions, or situations for which the motion measurements are erroneous, this first assimilation system would not give satisfying results. During an occlusion, it is mandatory that the unobserved curve's motion fields are inferred on the basis of some evolution law. In other words, the curve's motion field must also be tracked. To this end, an extended state vector gathering both a motion field and a curve representation must be considered and assimilated. This is the topics of the following part of the paper.

\section{Joint Assimilation of Motions and Curves}

The goal of this section is to show how the previous assimilation system can be enriched to handle the coupled tracking of a target curve and its motion. The idea consists in augmenting the state variable with a motion representation. To that end, an appropriate motion conservation law and motion measurements must also be added to the overall system. Two very different cases can be distinguished: fluid motions and natural objects motions. As a matter of fact, fluid motions are ruled by known and precise dynamical laws and concern situations for which the tracked curve does not delineate a specific object with its own motion. Fluid motion is a global entity that is almost independent from the curve location. This case corresponds therefore to a weakly coupled assimilation problem. The case of motions of natural objects in video is completely different. In this case, trajectories may change abruptly and the object's dynamics can 
not be described by generic physical models. As the scene background and the object to track have their own motions, the curve delineating the target object is usually the place of strong motion discontinuities. A good estimation of the object motion or of the background motion is-at least in the vicinity of the object frontier-essential. The quality of such estimation highly depends on the curve location. In this second case we thus have to tackle a tightly coupled assimilation problem.

These two cases will be studied separately. In the following section, we will first focus on the fluid motion case.

\subsection{Fluid Motion Images}

In several domains, the analysis of image sequences involving fluid phenomenon is of the highest importance. Such analysis is particularly meaningful as image sensors have the advantage of being noninvasive and provide almost dense spatio-temporal observation of the flow. Satellite images of the atmosphere and oceans are of particular interest in geophysical sciences such as meteorology and oceanography where one wants to track cloud systems, estimate ocean streams or monitor the drift of passive entities such as icebergs or pollutant sheets. The analysis of fluid flow images is also crucial in several domains of experimental fluid mechanics for the analysis of peculiar experimental flows or for flow control purposes. For all the kinds of aforementioned applications and domains, it is of major interest to track features transported by the flow along time. Such a tracking which consists in estimating Lagrangian drifters for the structures of interest may be obtained from deterministic integration methods such as the Euler method or the Runge and Kutta integration technique. These numerical integration approaches rely on a continuous spatio-temporal vector field description and thus require the use of interpolation schemes over the whole spatial and temporal domain of interest. As a consequence, they are quite sensitive to local errors measurements or to inaccurate motion estimates. When the images are noisy or if the flow velocities are of high magnitude and chaotic as in the case of turbulent flows, motion estimation tends to be quite difficult and prone to errors. Another source of error is inherent to motion estimation techniques (see for instance [3] for an extended review on motion estimation techniques). As a matter of fact, most of the motion estimation approaches use only a small set of images (usually two consecutive images of a sequence) and thus may suffer from a temporal inconsistency from frame to frame. The extension of spatial regularizers to spatio-temporal regularizers or the introduction of simple dynamical constraints in motion segmentation techniques relies mainly on crude stationary assumptions [1, $9-11,18,48,59]$.

Dedicated fluid motion estimators introduce adequate data terms [14, 15], relevant basis functions [21] or higher order regularizers $[15,29,61]$, in order to accurately estimate motion flows. However, these approaches do not include any dynamical constraint encouraging the preservation of conservation laws. Nothing warrants the solutions from being inconsistent with respect to the physical dynamical laws that the flow must obey.

Recently, motion estimation techniques relying on the vorticity-velocity formulation of Navier-Stokes equations have been proposed. In these works, the current solution is integrated forward in time with respect to this vorticity conservation law, in order to provide a new data term at the next frame [25, 26, 53]. In the same way as traditional curve tracking technique based on a propagation process, these methods do not guaranty a global dynamical consistency of the motion estimates. If at any frame, the estimation fails to provide, for some reasons, a result that is sufficiently close to the true motion field, the process may completely diverge. In that case, the result at a particular time is not driven by a consistent motion trajectory on a sufficiently large time range.

As we will show it in the following section, the inclusion within the variational assimilation framework of some conservation laws combined with crude motion observation enables to impose a global dynamical consistency and notably improve the quality of the recovered dense motion fields.

Fluid Definitions and Relations For fluid flows, the NavierStokes equation provides a universal general law for predicting the evolution of the flow. In this work, the formulation of the Navier-Stokes on which we will rely on, uses the vorticity $\xi$ and the divergence $\zeta$ of a motion field $\boldsymbol{w}=[u, v]^{T}$ :

$$
\begin{aligned}
& \xi=\nabla^{\perp} \cdot \boldsymbol{w}=\frac{\partial v}{\partial x}-\frac{\partial u}{\partial y}, \\
& \zeta=\nabla \cdot \boldsymbol{w}=\frac{\partial u}{\partial x}+\frac{\partial v}{\partial y} .
\end{aligned}
$$

The vorticity is related to the presence of a rotating motion, whereas the divergence is related to the presence of sinks and sources in a flow. Assuming that $\boldsymbol{w}$ vanishes at infinity, ${ }^{2}$ the vector field is decomposed using the orthogonal Helmholtz decomposition, as a sum of two potential function gradients:

$w=\nabla^{\perp} \Psi+\nabla \Phi$.

The stream function $\Psi$ and the velocity potential $\Phi$ respectively correspond to the solenoidal and the irrotational part of the vector field $\boldsymbol{w}$. They are linked to the divergence and

\footnotetext{
${ }^{2}$ In order to restrict the computation to the image domain, a divergence and curl free global transportation component is removed from the vector field. This field is estimated on the basis of a Horn and Schunck estimator associated to a high smoothing penalty [15].
} 
vorticity maps through two Poisson equations: $\xi=\Delta \Psi, \zeta=$ $\Delta \Phi$. Expressing the solution of both equations as a convolution product with the Green kernel $G(x)=\frac{1}{2 \pi} \operatorname{Ln}(|x|)$ associated to the 2D Laplacian operator:

$\Psi=G * \xi$
$\Phi=G * \zeta$,

the whole velocity field can be recovered knowing its divergence and vorticity:

$\boldsymbol{w}=\nabla^{\perp} G * \xi+\nabla G * \zeta \triangleq \boldsymbol{H}_{G}(\xi, \zeta)$.

This computation can be very efficiently implemented in the Fourier domain.

Fluid Motion Evolution Equation In order to consider a tractable expression of the Navier-Stokes equation for the tracking problem, we rely in this work on the incompressible vorticity-velocity formulation of the Navier-Stokes equation:

$\partial_{t} \xi+\boldsymbol{w} \cdot \nabla \xi=\nu_{\xi} \Delta \xi$

This formulation states that the vorticity is transported by the velocity field and diffuses along time. For the divergence map, it is more difficult to explicit any conservation law. We will assume that it behaves like a noise. More precisely, we will consider that the divergence map is a function of a Gaussian random variable, $\boldsymbol{X}_{t}$, with stationary increments (a Brownian motion) starting at points, $\boldsymbol{x}$, of the grid where the initial velocity field admits a nonnull divergence. It can be shown through Ito formula and Kolmogorov's backward equation, that the expectation at time $t$ of such a function, $u(t, \boldsymbol{x})=\mathbb{E}\left[\zeta\left(\boldsymbol{X}_{t}\right) \mid \boldsymbol{X}_{0}=\boldsymbol{x}\right]$ obeys a heat equation [44]:

$\partial_{t} u-v_{\zeta} \Delta u=0$,

$u(0, \boldsymbol{x})=\zeta(\boldsymbol{x})$.

According to this equation, we indeed make the assumption that the expectation of the divergence at any time of the sequence is a solution of a heat equation (i.e. it can be recovered from a smoothing of the initial motion field divergence map with a Gaussian function of standard deviation $2 \sqrt{v_{\zeta}} t$ ). The curl and divergence maps completely determine the underlying velocity field. Combining the motion equations (42) and (43) with the curve evolution law (33) proposed in the previous section leads to a complete dynamical model for the state function $X=[\phi, \xi, \zeta]$ gathering motions and curve descriptors, up to a control function $\boldsymbol{v}$ :

$\partial_{t}\left[\begin{array}{l}\phi \\ \xi \\ \zeta\end{array}\right]+\left[\begin{array}{c}\nabla \phi \cdot \boldsymbol{w}-\varepsilon\left(\Delta \phi-\frac{\nabla^{T} \phi H(\phi) \nabla \phi}{\|\nabla \phi\|^{2}}\right) \\ \boldsymbol{w} \cdot \nabla \xi-v_{\xi} \Delta \xi \\ -v_{\zeta} \Delta \zeta\end{array}\right]=\boldsymbol{v}$.
The first component of this evolution law is discretized in the same way as before. The vorticity-velocity equation is discretized following a total variation diminishing (TVD) scheme proposed in $[30,31]$ and associated to a third order Runge-Kutta integration [55]. The divergence equation is integrated through a stable implicit scheme. The motion field is updated at each time step in the Fourier domain with equation (41). Through this whole non-oscillatory scheme, the vorticity-velocity and divergence equations can be efficiently simulated.

Fluid Flows Observations As fluid motions are assumed to be continuous and defined over the whole image domain, we will assume that an observation motion field $\boldsymbol{w}_{o b s}$ is available. This motion field can be provided by any dense motion estimator. In this work, a dense motion field estimator dedicated to fluid flows is used [15]. Referring to equation (41), the observation operator for the motion components $\mathbb{H}(\boldsymbol{w})$ is defined as $\boldsymbol{w}_{o b s}=\boldsymbol{H}_{G}(\zeta, \xi)$. This operator links the motion measurements $\boldsymbol{w}_{o b s}$ to the state variable $\chi=[\zeta, \xi]^{T}$ (where $\chi$ is the compact notation for the div-curl coordinates). The observation equation for the motion components finally reads $Y(\chi)=\boldsymbol{H}_{G}(\chi)$. The observation operator is linear w.r.t $\chi$ so its linear tangent operator is itself. Nevertheless, as this operator is expressed through a convolution product, the expression of its adjoint $\partial_{\chi} \boldsymbol{H}_{G}^{*}$ reads [46]:

$\partial_{\chi} \boldsymbol{H}_{G}^{*}(\boldsymbol{w})=-\boldsymbol{H}_{G}(\boldsymbol{w})$.

The different covariance matrices, corresponding to the motion components, involved in the dynamic, in the initialization and in the observation equations, have been fixed to diagonal matrices with constant values (typically $B=0.1$ and $R=10$ ). As the dynamic is assumed to be quite accurate, the model covariance has been fixed to a low value of $Q=0.005$ for all the fluid image sequences. As the computational cost of this process is smaller than the optical flow computation on the whole sequence, it can be seen as a postprocessing of fluid dense motions. Otherwise, as the system we settled for the fluid motion case is weakly coupled, the assimilation of the curve and motion components do not have to be done simultaneously. Since the motion components do not depend on the curve components, they are first assimilated. Once the sequence of velocity fields have been analyzed, the curves are assimilated with their own observations.

\subsection{Natural Object Tracking}

As explained in the introduction of previous section, the situation of natural objects either rigid or deformable is completely different. Indeed, the curve and its motion are tightly coupled: we have to deal with an interlaced problem which can only be solved in a joint way. 
No universal physical law can be stated for natural objects observed from videos. Therefore, it is always a high difficulty in tracking applications to design a general law describing their evolution with accuracy. General rough models such as constant velocity or constant acceleration dynamic models are commonly used [27, 42]. This work also relies on that kind of models. Indeed, by assimilating the velocity on the whole sequence, we are going to reconstruct a time-consistent trajectory of the motion. We consider two kinds of motion representation: a dense motion model related to the whole image and an affine velocity directly linked to the trajectory of the tracked object. Nevertheless, let us note that a compromise solution between simple affine model and dense flow field could be defined on the basis of over-parameterized model as defined in [42].

\subsubsection{Dense Motion}

Evolution Law for Dense Motion Field We will assume that the targeted object moves with a constant velocity, up to an additive control function, along its trajectory. In differential form this reads:

$\frac{d \boldsymbol{w}}{d t}=\boldsymbol{v}_{\boldsymbol{w}}$.

As $w=[u, v]^{T}$, the evolution law for the dense motion fields is:

$\left\{\begin{array}{l}\partial_{t} u+\nabla u \cdot \boldsymbol{w}=v_{u}, \\ \partial_{t} v+\nabla v \cdot \boldsymbol{w}=v_{v},\end{array}\right.$

where $v_{u}$ and $v_{v}$ are control functions whose associated covariances are set to constant diagonal matrices. As the dynamic is here very likely approximative we used on the different sequence involved a value of $Q_{u}=Q_{v}=0.5$ for these coefficients. Combining the previous evolution law of the curve component (33) and the motion dynamic (46) leads to a coupled evolution model for the state function $[\phi, u, v]^{T}$ :

$\partial_{t}\left[\begin{array}{l}\phi \\ u \\ v\end{array}\right]+\left[\begin{array}{c}\boldsymbol{\nabla} \phi \cdot \boldsymbol{w}-\varepsilon\left(\Delta \phi-\frac{\nabla^{T} \phi H(\phi) \nabla \phi}{\|\nabla \phi\|^{2}}\right) \\ \nabla u \cdot \boldsymbol{w} \\ \nabla v \cdot \boldsymbol{w}\end{array}\right]=\boldsymbol{v}$.

To estimate the whole trajectory of the velocity field, it is necessary to define appropriate motion measurements.

Dense Motion Observations Accurate dense motion observations may be provided by an optical flow technique at each frame of the sequence. Nevertheless, in case of occlusions, these motion measurements do not describe the tracked object trajectory and must not be taken into account. We describe in the following the mechanism on which we resort to properly assimilate a complete trajectory of velocity fields. Assuming that the whole object is visible on the two first frames, the initial velocity field estimated between these two frames constitutes a good representation of the initial object's motion. In the following frames, in order to only consider velocity measurements related to the object of interest, it is necessary to define informative values for the covariance matrix associated to the motion measurements. High confidence values must be set at points where the object is visible (and low values where it is hidden). To fix these values, two distinct cases depending on the kind of contours observation we are dealing with must be considered separately.

- Noisy Curve Observations Model: In such a measurements model (Sect. 3.3.1), noisy or possibly inaccurate curves are assumed to be available when the whole object or some part of it is visible (i.e. $Y_{o b s}(\boldsymbol{x}, t)<0$ ). This provides area of the image where the object is visible. The motion observation covariance matrices can then be defined through the level set surfaces corresponding to these observed curves:

$$
R(\boldsymbol{x}, t)=R_{\min }+\left(R_{\max }-R_{\min }\right) \mathbf{1}_{Y_{o b s}(\boldsymbol{x}, t) \geq 0 .} .
$$

Hence, the dense motion observations are preponderantly considered only in regions where the object is visible. In our applications, we systematically chose $R_{\min }=10$ and $R_{\max }=50$.

- Image Observation Model: When the measurements model relies on image local histograms (Sect. 3.3.2), the situation is more problematic as no curves are directly observed. We also want to avoid the use of an external segmentation process to infer occlusion situations. Considering the object and the background reference histograms $\left(\rho_{o}\right.$ and $\left.\rho_{b}\right)$, it is possible to define adequate confidence values for the motion observation covariance matrix. We propose to fix them as:

$$
R(\boldsymbol{x}, t)=R_{\min }+\left(R_{\max }-R_{\min }\right) \mathbf{1}_{d_{B}\left(\rho_{V_{x}}, \rho_{o}\right)<d_{B}\left(\rho_{V_{x}}, \rho_{b}\right)} .
$$

Thus, low values $\left(R_{\min }=5\right)$ are associated to points that likely belong to the background, whereas higher confidence values $\left(R_{\max }=20\right)$ are related to the other points.

\subsubsection{Affine Velocity}

In some cases, it is sometimes sufficient to rely on a reduced parametric representation of the object's motion. We now consider an affine velocity directly related to the target. In the following, we will assume that the apparent motion over the area delineated by the curve follows an affine parametric motion, i.e:

$\boldsymbol{w}(\boldsymbol{x}, t)=\boldsymbol{S}(t) \boldsymbol{x}+\boldsymbol{T}(t) \quad \forall \boldsymbol{x} \in \Omega \mid \phi(\boldsymbol{x})<0$,

where $\boldsymbol{S}(t)$ is the rotation, divergence and shear matrix and $\boldsymbol{T}(t)$ is a translation vector. 
Evolution Law for Affine Velocity Assuming that the targeted object move with a constant velocity, up to a control function, along its trajectory, as defined in equation (45), we obtain:

$$
\begin{aligned}
& \left(\partial_{t} \boldsymbol{S}(t)+\boldsymbol{S}^{2}(t)\right) \boldsymbol{x}+\partial_{t} \boldsymbol{T}(t)+\boldsymbol{S}(t) \boldsymbol{T}(t)=\boldsymbol{v}_{\boldsymbol{w}} \\
& \quad \forall \boldsymbol{x} \in \Omega \mid \phi(x)<0 .
\end{aligned}
$$

As this equation must be respected for all points inside the object, we get the following system of equations:

$$
\left\{\begin{array}{l}
\partial_{t} \boldsymbol{S}(t)+\boldsymbol{S}^{2}(t)=\boldsymbol{v}_{S}, \\
\partial_{t} \boldsymbol{T}(t)+\boldsymbol{S}(t) \boldsymbol{T}(t)=\boldsymbol{v}_{T},
\end{array}\right.
$$

where $\boldsymbol{v}_{S}$ and $\boldsymbol{v}_{T}$ are control functions with constant diagonal covariance matrices (we typically chose $Q_{S}=Q_{T}=$ $0.01)$. If $\boldsymbol{S}^{-1}\left(t_{0}\right)$ exists, a closed form solution of the system of equations (51) without second members exists:

$$
\left\{\begin{array}{l}
\boldsymbol{S}(t)=\left(\boldsymbol{S}^{-1}\left(t_{0}\right)-\boldsymbol{I d} t\right)^{-1}, \\
\boldsymbol{T}(t)=\boldsymbol{S}(t) \boldsymbol{T}^{-1}\left(t_{0}\right) \boldsymbol{T}\left(t_{0}\right) .
\end{array}\right.
$$

This solution can be readily used for the first forward integration of the motion components in the assimilation process. Combining the curve evolution law (33) and the previous motion dynamical law (51) leads to a coupled evolution model for the state function $[\phi, S, T]^{T}$ :

$\partial_{t}\left[\begin{array}{c}\phi \\ \boldsymbol{S} \\ \boldsymbol{T}\end{array}\right]+\left[\begin{array}{c}\boldsymbol{\nabla} \phi \cdot \boldsymbol{w}-\varepsilon\left(\Delta \phi-\frac{\nabla^{T} \phi H(\phi) \nabla \phi}{\|\nabla \phi\|^{2}}\right) \\ \boldsymbol{S}^{2}(t) \\ \boldsymbol{S}(t) \boldsymbol{T}(t)\end{array}\right]=\boldsymbol{v}$.

Let us now explain the different measurements $Y_{S}$ and $Y_{T}$ associated to this evolution law.

Affine Velocity Observations As for the dense motion model, it is essential to have some significant motion measurements at one's disposal. Two measurement models should still be distinguished.

- Noisy Curve Observations Model: For the measurements model (Sect. 3.3.1), the observed contours enable to perform a robust parametric motion estimation inside the region of interest. Such a process directly provides the components of the observation functions, $Y_{S}$ and $Y_{T}$. The values of the least square residuals are used to fix the corresponding covariance values [43]. When no curves are provided or if the area delineated is to small, no motion estimations are considered and the corresponding values of the observation covariance matrix are set to infinity.

- Image Observation Model: When the measurements model relies directly on image local histograms (Sect. 3.3.2), the only available region on which it is possible to get motion estimates is the one specified as the initial condition at the initial time. This single rough parametric motion measurement reveals to be generally unable to anticipate sufficiently well the object's trajectory on the whole sequence during the first forward step. For such a measurement model, we have specifically considered a final condition on the object contour as an additional equation of the assimilation system (see Sect. 2.3). Assuming that the object is visible (at least partially) both on the initial and final image of the sequence, motion measurements associated to these two object locations allow us to assimilate roughly as a first guess the object motion. Latter on, the current sequence of curve estimates provide support to run a robust parametric estimation and thus enable to refine the motion measurements. More precisely, the motion estimation is run only on points of the support region associated to high confidence covariance values. The covariance values are fixed in the same way as in (48). Such a recipe avoids to perform motion estimation on occluded parts of the target.

Let us remark that the computational cost of the affine velocity assimilation is very small in comparison with the dense motion case. Indeed, the motion state variables are only $(2 \times 2)$ matrix and $(1 \times 2)$ vector, so the assimilation process for affine motion is instantaneous. Nevertheless, the computational cost of a dense velocity field assimilation remains smaller than the computation of the optical flow on the whole image sequence.

\subsection{Results}

Fluid Motion In order to assess the benefits of fluid motion assimilation we first applied the motion assimilation without curve tracking on a synthetic sequence of particles images of 2D turbulence obtained through a direct numerical simulation (DNS) of the Navier-Stokes equation [12]. For this sequence composed of 50 images, we compare in Fig. 9 the vorticity map of respectively the actual, the observed and the assimilated motion fields.

We can see that the assimilation not only denoises the observations, but also allows some small scales structures to be recovered thanks to the vorticity-velocity dynamical model. The motion measurements have been provided by a dedicated optical flow estimator [15]. This estimator has been thoroughly used on experimental flows [14] and has demonstrated to give much better results than traditional optical flow schemes for fluid sequence. It allows in particular a much more accurate estimation of vorticity and divergence compared to state of the art PIV correlation techniques.

To give some quantitative evaluation results, we present in Fig. 10 comparative errors between the ground truth and the obtained results. On this example, the assimilation 
Fig. 9 2D direct numerical simulation. (a) Particle images sequence. (b) True vorticity. (c) Vorticity observed by the optical flow estimator. (d) Assimilated vorticity a)

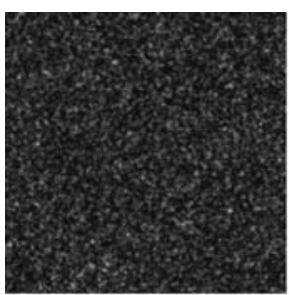

b)

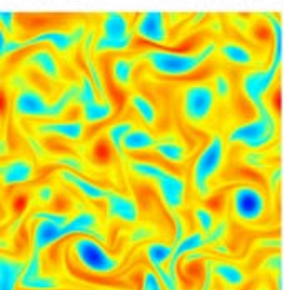

c)

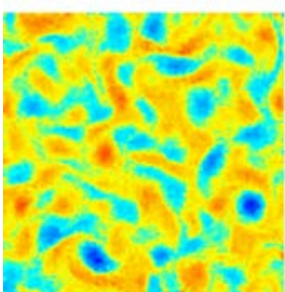

d)

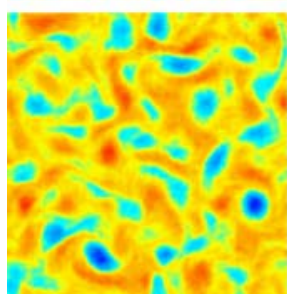

$\mathrm{t}=10$
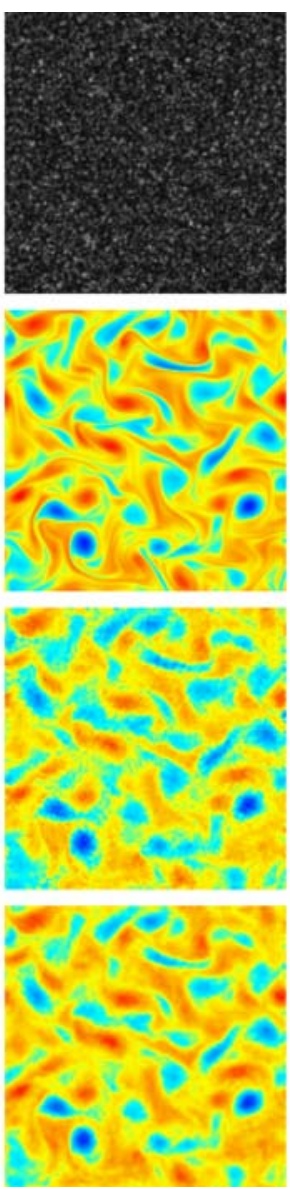

$t=30$
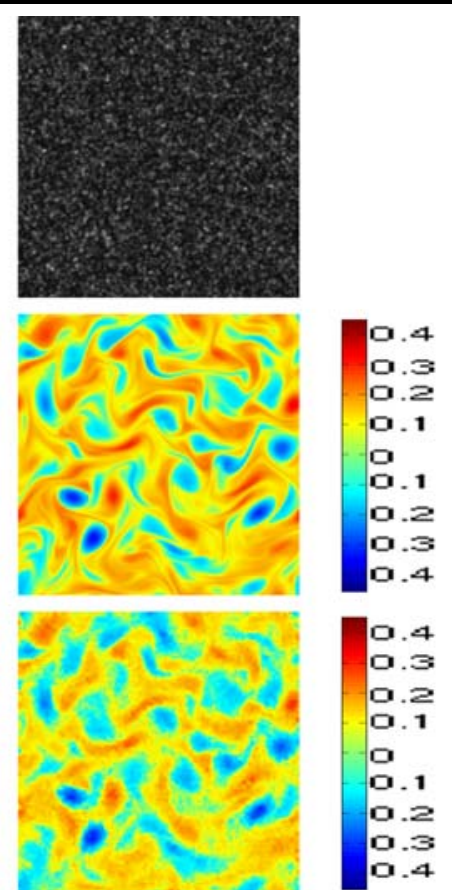

0.4

0.3

0.2

a. 1

a

a. 1

0.2

0.3

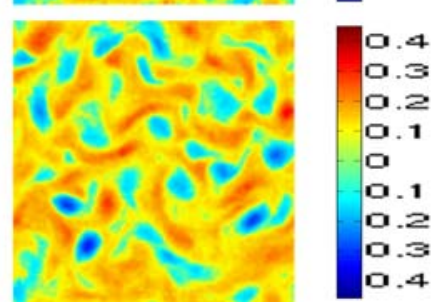

$\mathrm{t}=50$

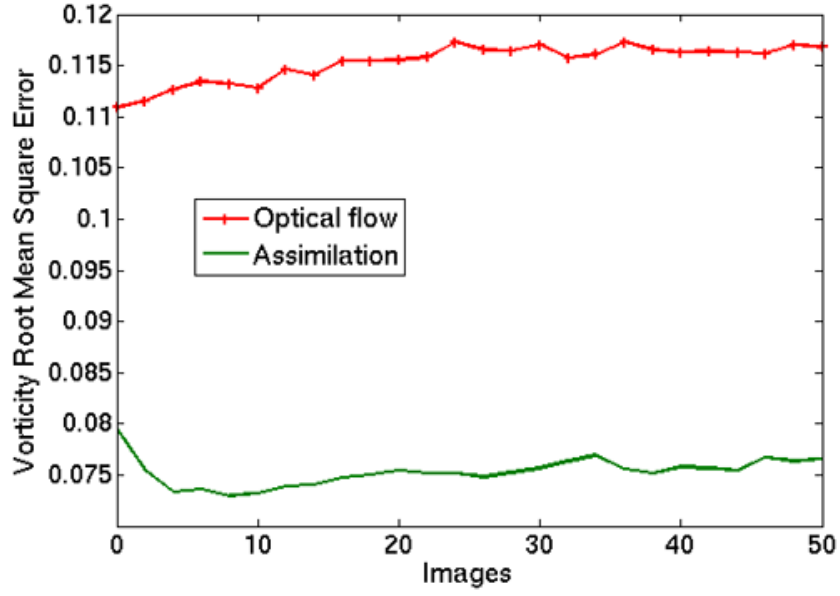

Fig. 10 Comparison of errors. The upper curve outlines the mean square error of the vorticity computed by the optical flow technique dedicated to fluid flows [15] on the 50 images of the turbulent particles image sequence. The lower curve exhibits the error obtained at the end of the assimilation process. The assimilated vorticity is then closer to the reality than the observed one

process enables to significantly improve the quality of the recovered motion field (about 30\%).
To demonstrate the usefulness of the joint tracking of curve and fluid motion, we apply the complete assimilation process on Infra-red meteorological sequences showing hurricanes. The first sequence corresponds to a cyclone observed over the Indian Ocean. The second one shows the cyclone Vince observed over north Atlantic. In these two experiments, the motion fields obtained by the dedicated fluid motion estimator $[14,15]$ are first assimilated with the process presented in Sect. 4.1. The user then selects a curve of interest on the first image and the curve is assimilated with the curve observation equation given in Sect. 3.3.2.

The Fig. 11 shows for the cyclone over the Indian Ocean the curve location on the final image of the sequence obtained considering respectively the motion fields as a deterministic inputs of the system (as in the first part of this paper) and considering a joint assimilation of fluid motion. As it can be observed from the location of the curve on the last image of the sequence, the joint curve and motion assimilations enables to recover a curve location that better fits the hurricane. The iso-temperature curve is much better tracked along time when considering a coupled tracking.

Complete results in term of curves and vorticity maps are presented on Fig. 12 for the cyclone over the Indian Ocean. 
Fig. 11 Final position of the curve on the 19th frame. Left: Initialization at the first frame. Middle: Final curve position without fluid motion assimilation. Right: Final curve position with a joint fluid motion assimilation

Fig. 12 Cyclone sequence. (a) Sample of observed curves. (b) Sample of observed vorticity maps. (c) Recovered curves.

(d) Vorticity maps corresponding to the recovered motion fields

Fig. 13 Vince cyclone infra-red sequence. Result of the assimilation technique with the photometric measurement model based on the local probability densities
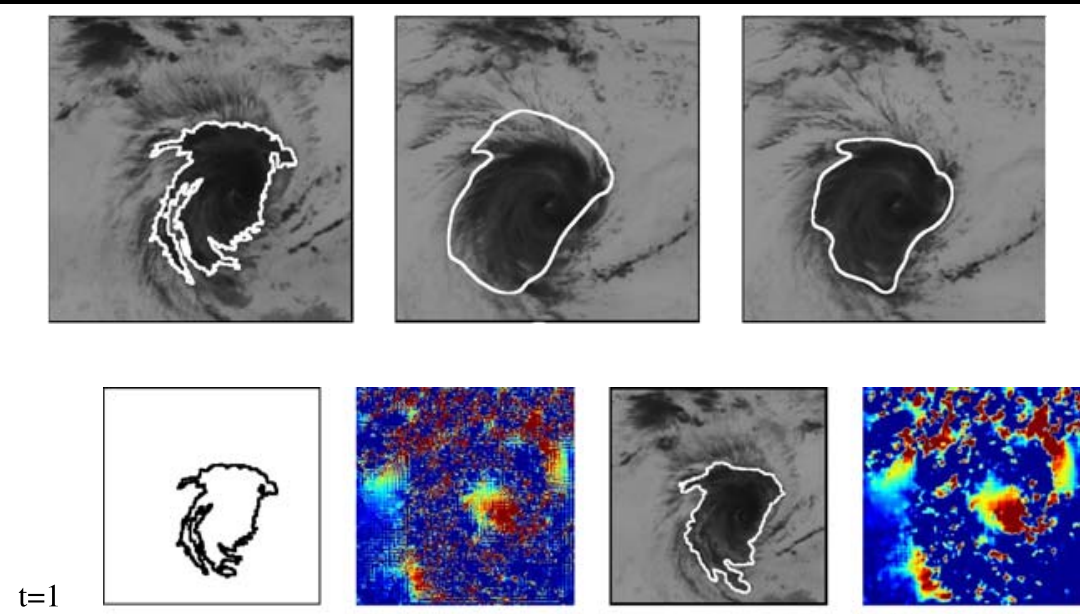

NO OBSERVATIONS
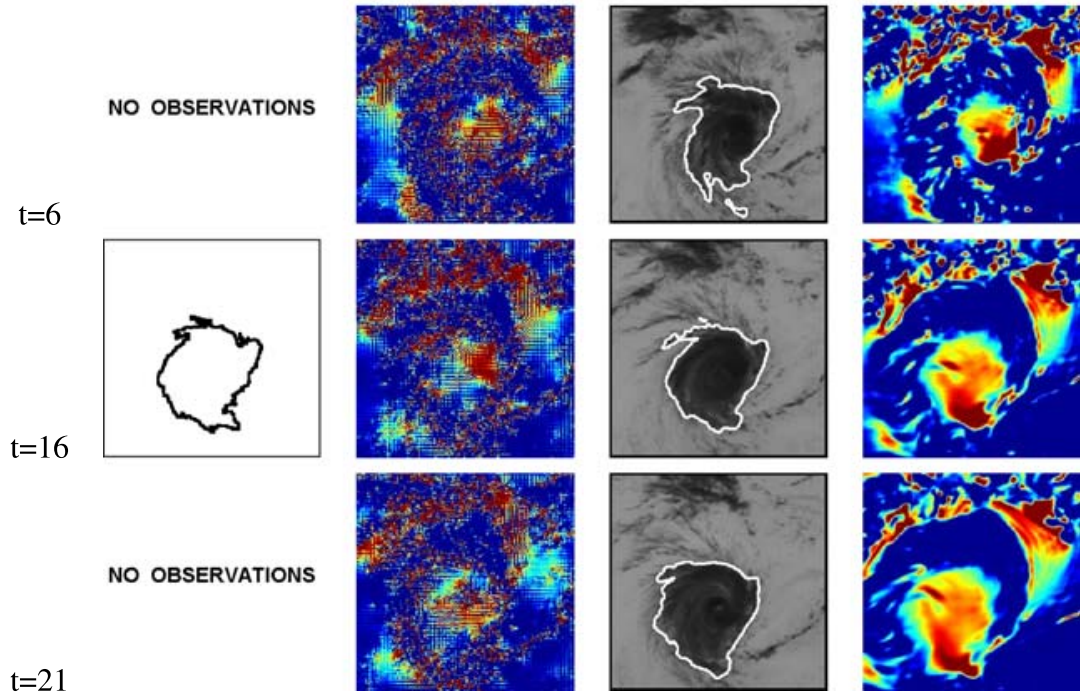

(a)

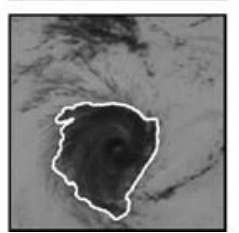

(c)

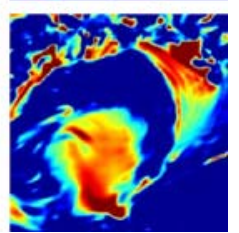

(b)

(d)

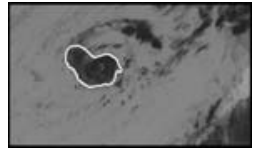

$t=0$

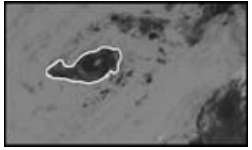

$t=12$

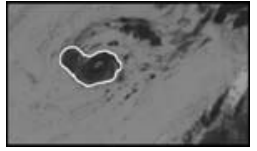

$t=3$

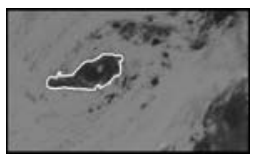

$t=14$

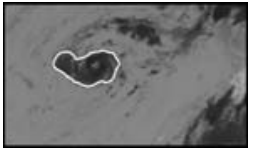

$t=6$

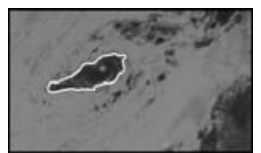

$t=16$

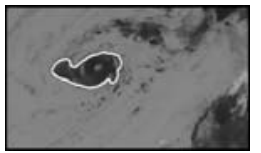

$t=9$

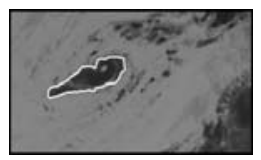

$t=18$
Column (a) shows the different photometric level lines used as curve measurements, whereas column (b) exhibits the different vorticity maps of the initial motion fields used as motion measurements. As it can be observed from the plotted vorticity maps, these measurements constitute very noisy motion observations for the system we want to track. As we used a fast motion estimator, the motion observations present a lot of temporal inconsistencies with non plausible discontinuities artifacts. The photometric level-lines are also quite noisy. To further demonstrate the ability of the proposed method, these photometric level lines have not been extracted for each image (these level lines have been extracted only every three images along the sequence). The estimated curve superimposed on the initial image sequence and the recovered vorticity maps are respectively shown on columns (c) and (d). We can notice that the motion fields recovered after assimilation are much more coherent from a physical point of view. 


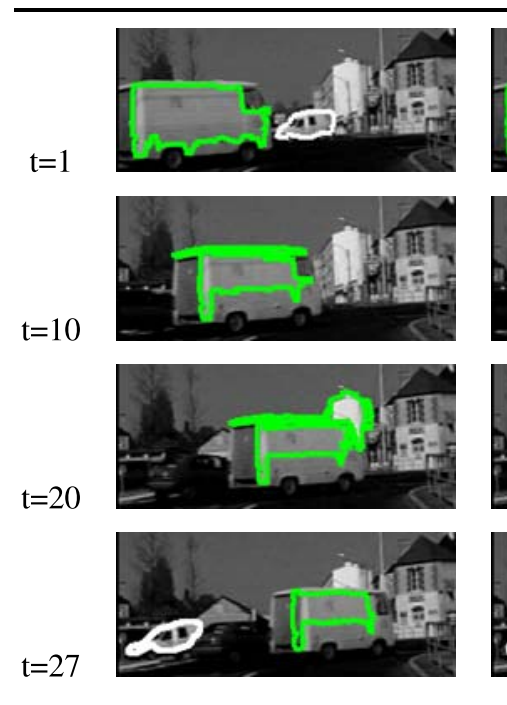

(a)

Fig. 14 Car sequence 2. (a) Sample of observed photometric level lines. (b) Curves after joint assimilation

The results presented in Fig. 13 show the final curves recovered for the Vince cyclone. This sequence is composed of 19 images obtained on the 9th of October 2005 from $00 \mathrm{H} 15$ to $4 \mathrm{H} 45 .{ }^{3}$ For this image sequence, we rely on the local photometric distributions measurement model. That is to say we aim at tracking here a temperature distribution of reference defined from an initial mask on the first image. The joint assimilation of motion and curve enables to track perfectly the cyclone.

Natural Objects To demonstrate the potential benefit of a joint assimilation of closed curves and their motions for natural video sequences, we show results on two sequences exhibiting long term total occlusions.

As a first result we show the tracking of two curves representing the photometric level lines of two cars in a video sequence of 27 frames. As can be observed, one of the cars is occluded by the other during a long time period. In this case, we rely on an affine parametrization (Sect. 4.2.2) to describe the objects motion. We assume that some observed curves are available, so we here refer to the curve observation model given in Sect. 3.3.1. The motion measurements are given by motion estimates within the areas delineated by the observed curves [43] when they are available. Let us precise that, in this example, a final target is considered for the motion assimilation. The level lines corresponding to the curve measurements are shown on the column (a) of the Fig. 14. Obviously, there is neither curve nor motion measurements corresponding to the white car during its occlusion. Two level functions (one for each object) have been

\footnotetext{
${ }^{3}$ We thank the Laboratoire de Météorologie Dynamique for providing us this sequence.
}

used in this example. Column (b) of Fig. 14 present the curves recovered for the two cars. As can be observed these level lines are of bad quality and do not really describe a precise boundary of the objects of interest. Let us note that even if the white car does not have a constant velocity during the occlusion, the assimilation process enables to predict quite well its photometric level line.

For the second experiment we present results obtained on a sequence of 22 frames showing a surfer into a water pipeline. In this sequence, the surfer, our target object, disappears into the pipeline during 15 images. The target is therefore not visible during $70 \%$ of the sequence. For this sequence we have used the local photometric probability distribution measurements described in Sect. 3.3.2. The reference distribution of the surfer is deduced from an initial curve obtained through the selection of a given photometric level line. This first initial curve which only describes inaccurately the surfers contours is presented on Fig. 15. Dense motion fields have been computed through an optical flow estimator on each frame of the sequence. These motion measurements have been assimilated with a constant velocity dynamical model and an image adapted covariance matrix as described in Sect. 4.2.1. The recovered curves are presented in Fig. 15. As can be observed, the joint tracking of a curve and of its motion enables to recover a coherent position of the surfer during the whole sequence. Let us precise that, in this example, no final target has been given by the user. This example exhibits the ability of the proposed method to use the complete set of measurements to provide a global reconstruction of the trajectory of a curve and of its motion.

\section{Conclusion}

In this paper, a new framework allowing a coupled tracking of curves and vector fields has been presented. The proposed approach is related to variational data assimilation technique and allows the batch mode reconstruction of the trajectory of features leaving in spaces of very large dimension.

In a similar way to a stochastic smoothing, the estimation is led considering the whole set of available measurements extracted from the image sequence. The technique is nevertheless totally different. It consists in integrating two coupled pde's representing the evolution of a state function and its adjoint variable. The method incorporates only few parameters. Similarly to Bayesian filtering techniques, these parameters mainly concern the definition of the different error models involved in the considered system.

For the tracking of natural objects in video, the main difficulty concerns the velocities. If no coherent motion can be computed on the whole sequence as a first guess, the tracking algorithm can fail. Actually, a solution could be to rely 
Fig. 15 Surfer into a water pipeline. The only curve available is the initialization, shown on the first image of the figure. The tracking results are presented for different frames of this 22 frames sequence

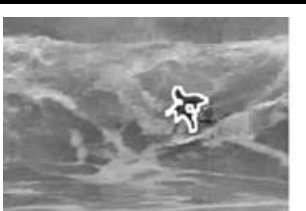

Initialization

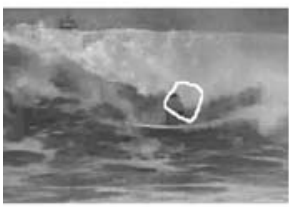

$\mathrm{t}=4$

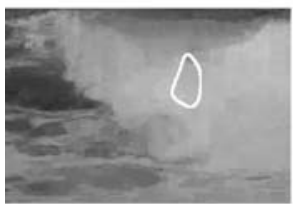

$\mathrm{t}=12$

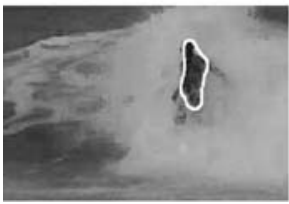

$\mathrm{t}=19$

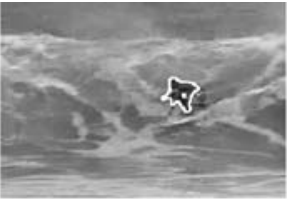

$\mathrm{t}=0$

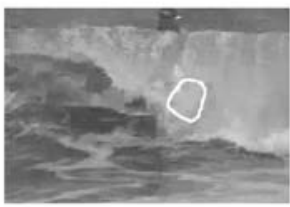

$\mathrm{t}=6$

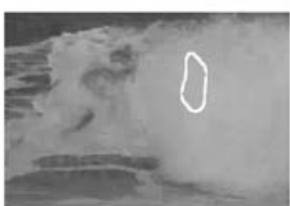

$\mathrm{t}=15$

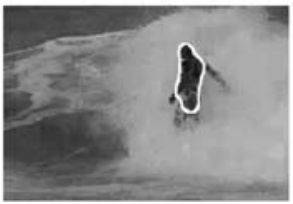

$\mathrm{t}=20$

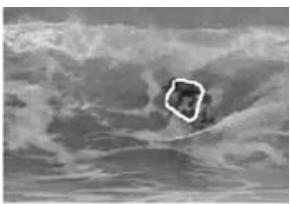

$\mathrm{t}=2$

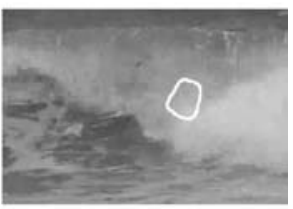

$\mathrm{t}=9$

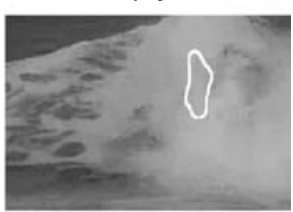

$\mathrm{t}=18$

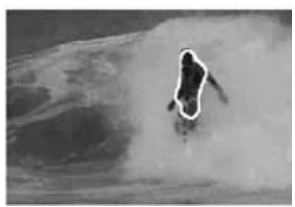

$\mathrm{t}=21$ on particle filtering, in order to find a first coherent motion. In fluid motion cases, the proposed technique gives very good results. It enables to enforce the motion field to respect some physical conservation and improve consequently the consistency of the solution.

Concerning applications, we believe that the proposed assimilation process is of major interest when one aims at analyzing the deformations along time of entities enclosed by a curve. For instance, such an analysis is informative in environmental sciences for analyzing, representing or understanding complex phenomena. These phenomena may be only partially observable and subject to occlusions. In video analysis, such a framework could be interesting for video post-processing of moving objects, such as object removal, object based video editing, off-line structuring, or indexing of video databases.

Acknowledgements This work was supported by the European Community through the IST FET Open FLUID project (http: // fluid.irisa.fr).

\section{References}

1. Amiaz, T., Kiryati, N.: Piecewise-smooth dense optical flow via level sets. Int. J. Comput. Vis. 68(2), 111-124 (2006)

2. Anderson, B., Moore, J.: Optimal Filtering. Prentice Hall, Englewood Cliffs (1979)

3. Barron, J., Fleet, D., Beauchemin, S.: Performance of optical flow techniques. Int. J. Comput. Vis. 12(1), 43-77 (1994)
4. Bartesaghi, A., Sapiro, G.: Tracking of moving objects under severe and total occlusions. In Proc. Int. Conf. Image Processing, ICIP'05, vol. 1, pp. 301-304 (2005)

5. Beg, M., Miller, M., Trouvé, A., Younes, L.: Computing large deformation metric mappings via geodesic flows of diffeomorphisms. Int. J. Comput. Vis. 61(2), 139-157 (2005)

6. Bennet, A.: Inverse Methods in Physical Oceanography. Cambridge University Press, Cambridge (1992)

7. Blake, A., Isard, M.: Active Contours. Springer, London (1998)

8. Bouttier, F., Courtier, P.: Data Assimilation Concepts and Methods. ECMWF Meteorological Training Course Lecture Series (1999)

9. Brox, T., Bruhn, A., Papenberg, N., Weickert, J.: High accuracy optical flow estimation based on a theory for warping. In: Proc. Eur. Conf. Comput. Vis., ECCV'04, Prague, Czech Republic, pp. 25-36. Springer, Berlin (2004)

10. Brox, T., Bruhn, A., Weickert, J.: Variational motion segmentation with level sets. In Proc. Eur. Conf. Comput. Vis., vol. 1, pp. 471483 (2006)

11. Bruhn, A., Weickert, J., Schnörr, C.: Lucas/Kanade meets Horn/Schunck: combining local and global optic flow methods. Int. J. Comput. Vis. 61(3), 211-231 (2005)

12. Carlier, J., Wieneke, B.: Report on production and diffusion of fluid mechanics images and data. Technical report, Fluid Project deliverable 1.2 (2005)

13. Chan, T., Vese, L.: Active contours without edges. IEEE Trans. Image Process. 10(2), 266-277 (2001)

14. Corpetti, T., Heitz, D., Arroyo, G., Mémin, E., Santa-Cruz, A.: Fluid experimental flow estimation based on an optical-flow scheme. Int. J. Exp. Fluid 40(1), 80-97 (2006)

15. Corpetti, T., Mémin, E., Pérez, P.: Dense estimation of fluid flows. IEEE Trans. Pattern Anal. Mach. Intell. 24(3), 365-380 (2002)

16. Courtier, P., Talagrand, O.: Variational assimilation of meteorological observations with the direct and adjoint shallow-water equations. Tellus 42, 531-549 (1990) 
17. Cremers, D.: Dynamical statistical shape priors for level set based tracking. IEEE Trans. Pattern Anal. Mach. Intell. 28(8), 1262$1273(2006)$

18. Cremers, D., Soatto, S.: Variational space-time motion segmentation. In: Proc. IEEE Int. Conf. Comput. Vis. ICCV'03, vol. 2, pp. 886-892 (2003)

19. Cremers, D., Soatto, S.: Motion competition: a variational framework for piecewise parametric motion segmentation. Int. J. Comput. Vis. 62(3), 249-265 (2005)

20. Cremers, D., Tischhäuser, F., Weickert, J., Schnoerr, C.: Diffusion snakes: introducing statistical shape knowledge into the MumfordShah functional. Int. J. Comput. Vis. 50(3), 295-313 (2002)

21. Cuzol, A., Hellier, P., Memin, E.: A low dimensional fluid motion estimator. Int. J. Comput. Vis. 75(3), 329-349 (2007)

22. Dambreville, S., Rathi, Y., Tannenbaum, A.: Tracking deformable objects with unscented Kalman filtering and geometric active contours. In: American Control Conf., ACC'06, June 2006

23. Faugeras, B.: Assimilation variationnelle de données dans un modèle couplé océan-biogéochimie. $\mathrm{PhD}$ thesis, Université Joseph-Fourier-Grenoble I, Octobre 2002

24. Goldenberg, R., Kimmel, R., Rivlin, E., Rudzsky, M.: Fast geodesic active contours. IEEE Trans. Image Process. 10(10), 14671475 (2001)

25. Héas, P., Mémin, E., Papadakis, N., Szantai, A.: Layered estimation of atmospheric mesoscale dynamics from satellite imagery. IEEE Trans. Geosci. Remote Sens. 45(12), 4087-4104 (2007)

26. Héas, P., Papadakis, N., Mémin, E.: Time-consistent estimators of $2 \mathrm{~d} / 3 \mathrm{~d}$ motion of atmospheric layers from pressure images. Technical Report 6292, INRIA, September 2007

27. Jackson, J., Yezzi, A., Soatto, S.: Tracking deformable moving objects under severe occlusions. In: IEEE Conference on Decision and Control, December 2004

28. Kimmel, R., Bruckstein, A.M.: Tracking level sets by level sets: a method for solving the shape from shading problem. Comput. Vis. Image Underst. 62(1), 47-58 (1995)

29. Kohlberger, T., Mémin, E., Schnörr, C.: Variational dense motion estimation using the Helmholtz decomposition. In: Proc. Int. Conf. on Scale-Space and PDE methods in Comput. Vis., ScaleSpace'03, Isle of Skye, UK, pp. 432-448 (2003)

30. Kurganov, A., Levy, D.: A third-order semidiscrete central scheme for conservation laws and convection-diffusion equations. SIAM J. Sci. Comput. 22(4), 1461-1488 (2000)

31. Kurganov, A., Tadmor, E.: New high-resolution central schemes for nonlinear conservation laws and convection-diffusion equations. J. Comput. Phys. 160(1), 241-282 (2000)

32. Le Dimet, F.-X., Talagrand, O.: Variational algorithms for analysis and assimilation of meteorological observations: theoretical aspects. Tellus 38(A), 97-110 (1986)

33. Leventon, M., Grimson, E., Faugeras, O.: Statistical shape influence in geodesic active contours. In: Proc. IEEE Comput. Vis. and Pat. Rec. CVPR'00 (2000)

34. Li, Z., Navon, I.M.: Optimality of 4d-var and its relationship with the Kalman filter and Kalman smoother. Q. J. R. Meteorol. Soc. 127(572), 661-683 (2001)

35. Lions, J.: Contrôle Optimal de Systèmes Gouvernés par des Équations aux Dérivées Partielles. Dunod, Paris (1968)

36. Lions, J.: Optimal Control of Systems Governed by PDEs. Springer, New York (1971)

37. Mansouri, A.: Region tracking via level set PDEs without motion computation. IEEE Trans. Pattern Anal. Mach. Intell. 24(7), 947961 (2003)

38. Martin, P., Réfrégier, P., Goudail, F., Guérault, F.: Influence of the noise model on level set active contours segmentation. IEEE Trans. Pattern Anal. Mach. Intell. 26(6), 799-803 (2004)

39. Mémin, E., Pérez, P.: Dense estimation and object-based segmentation of the optical flow with robust techniques. IEEE Trans. Image Process. 7(5), 703-719 (1998)
40. Niethammer, M., Tannenbaum, A.: Dynamic geodesic snakes for visual tracking. In: Proc. IEEE Comput. Vis. and Pat. Rec. CVPR'04, vol. 1, pp. 660-667 (2004)

41. Niethammer, M., Vela, P., Tannenbaum, A.: Geometric observers for dynamically evolving curves. In: Proc. Conf. Decision and Control Eur. Control Conf. CDC-ECC'05, pp. 6071-6077 (2005)

42. Nir, T., Bruckstein, A., Kimmel, R.: Over parameterized opticalflow. Technical Report CIS-2006-05, Technion-Israel Institute of Technology (2006)

43. Odobez, J.-M., Bouthemy, P.: Robust multiresolution estimation of parametric motion models. J. Vis. Commun. Image Represent. 6(4), 348-365 (1995)

44. Oksendal, B.: Stochastic Differential Equations. Springer, Berlin (1998)

45. Osher, S., Sethian, J.: Fronts propagating with curvature dependent speed: algorithms based on Hamilton-Jacobi formulation. J. Comput. Phys. 79, 12-49 (1988)

46. Papadakis, N., Mémin, E.: A variational method for joint tracking of curve and motion. Research Report 6283, INRIA, September 2007

47. Papadakis, N., Mémin, E.: Variational optimal control technique for the tracking of deformable objects. In: Proc. IEEE Int. Conf. Comput. Vis., ICCV'07, Rio de Janeiro, Brazil, October 2007

48. Papenberg, N., Bruhn, A., Brox, T., Didas, S., Weickert, J.: Highly accurate optic flow computation with theoretically justified warping. Int. J. Comput. Vis. 67(2), 141-158 (2005)

49. Paragios, N.: A level set approach for shape-driven segmentation and tracking of the left ventricle. IEEE Trans. Med. Imaging 22(6), 773-776 (2003)

50. Paragios, N., Deriche, R.: Geodesic active regions: a new framework to deal with frame partition problems in computer vision. J. Vis. Commun. Image Represent. 13, 249-268 (2002)

51. Peterfreund, N.: Robust tracking of position and velocity with Kalman snakes. IEEE Trans. Pattern Anal. Mach. Intell. 21(6), 564-569 (1999)

52. Rathi, Y., Vaswani, N., Tannenbaum, A., Yezzi, A.: Tracking deforming objects using particle filtering for geometric active contours. IEEE Trans. Pattern Anal. Mach. Intell. 29(8), 1470-1475 (2007)

53. Ruhnau, P., Stahl, A., Schnörr, C.: Variational estimation of experimental fluid flows with physics-based spatio-temporal regularization. Meas. Sci. Technol. 18(3), 755-763 (2007)

54. Sethian, J.: Level Set Methods. Cambridge University Press, Cambridge (1996)

55. Shu, C.-W.: Essentially non-oscillatory and weighted essentially non-oscillatory schemes for hyperbolic conservation laws. In: Advanced Numerical Approximation of Nonlinear Hyperbolic Equations. Lecture Notes in Mathematics, vol. 1697, pp. 325-432. Springer, Berlin (1998)

56. Soatto, S., Yezzi, A., Duci, A.: Region matching and tracking under deformations and occlusions. In: Geometric Level Sets Methods in Imaging Vision and Graphics. Springer, Berlin (2003)

57. Talagrand, O., Courtier, P.: Variational assimilation of meteorological observations with the adjoint vorticity equation, I: theory. Q. J. R. Meteorol. Soc. 113, 1311-1328 (1987)

58. Trémolet, Y.: Incremental 4d-var convergence study. Tellus A 59(5), 706-718 (2007)

59. Weickert, J., Schnörr, C.: Variational optic-flow computation with a spatio-temporal smoothness constraint. J. Math. Imaging Vis. 14(3), 245-255 (2001)

60. Yezzi, A., Zöllei, L., Kapur, T.: A variational framework for joint segmentation and registration. In: MMBIA, pp. 44-51 (2001)

61. Yuan, J., Schnörr, C., Mémin, E.: Discrete orthogonal decomposition and variational fluid flow estimation. J. Math. Imaging Vis. 28(1), 67-80 (2007) 


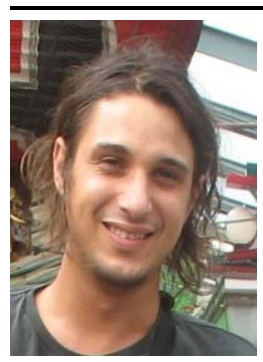

N. Papadakis was born in 1981. He graduated from the National Institute of Applied Sciences (INSA) of Rouen in Applied Mathematics, France, in 2004. He received the Ph.D. degree in Applied Mathematics fron the University of Rennes, France, in 2007. His main research interests are on variational methods for tracking in image sequences.

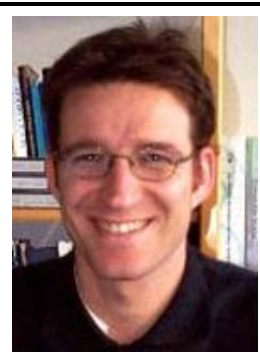

E. Mémin was born in 1965. He received the $\mathrm{Ph} . \mathrm{D}$. degree in Computer Science fron the University of Rennes, France, in 1993. He was an assistant professor at the University of Bretagne Sud, France fron 1993 to 1999. He now holds a position at the University of Rennes, France. His research interests include computer vision, statitical models for image (sequence) analysis, fluid and medical images, and parallel algorithms for computer vision. 